\title{
Stochastic Integrated manufacturing and Remanufacturing model with Shortage, Rework and Quality Based Return Rate in a Closed Loop Supply Chain
}

\begin{abstract}
Over the last few decades, inventory management of produced, recovered, and returned items in the closed loop supply chain (CLSC) systems has been significantly taken into consideration. Most of available works in the literature assumed that manufactured and remanufactured items have the same quality. However in practices, some customers perceive remanufactured items as lower quality products rather than newly produced items. Therefore, this paper assumes demands for manufactured items and remanufactured ones are not same. This assumption causes lost sales in which there are shortage periods for both produced and reproduced items. In the real-life production systems, the generation of nonconforming items is inevitable owing to deteriorating process or other uncontrollable factors. In order to take this issue into account, the production and reproduction processes are assumed to be imperfect as it can produce some defectives which would be reworked in the same cycles. Moreover, to make the study realistic, we assume the return rate of used items as a variable function which depends on the minimum acceptable quality level of returns. Furthermore, in this research the quality of returned items is considered as a random variable and based on this assumption, buyback cost, remanufacturing cost and salvage value depend on quality level of returned items. Basically, three different stochastic models, with different probability distribution functions, are mathematically developed for multiple manufacturing and remanufacturing cycles. A solution procedure is introduced and then there numerical studies are provided to show the applicability of the developed models and also to perform a sensitivity analysis. Finally, conclusion and future researches are presented.
\end{abstract}

Keywords: Closed-loop supply chain; Production; Remanufacturing; Lost Sales; Defective items; Rework Process.

\section{Introduction}

Inventory control is one of the most critical operations for any company and should be handled effectively to survive and promote in the fierce and competitive businesses. So, companies take serious measures to deal with their inventories, equipment, and spares. For exhaustive reviews, we refer the reader to Cárdenas-Barrón et al. [1].

Over the last few decades, recycling and remanufacturing have been receiving increasing attention due to the requirement of sustainable development and environmental concerns including, air pollution, waste disposal, and natural resource depletion. Environment consciousness forces companies to establish such recycling systems in which used product are collected from customers once they pass their economic or effective lives. Besides, the pressures from $\mathrm{WEEE}^{1}, \mathrm{EU}^{2}, \mathrm{RoHS}^{3}$ and $\mathrm{EuP}^{4}$ have significantly influence on the development of green products and saving natural resources for next generations, so companies can

\footnotetext{
${ }^{1}$ Waste Electrical and Electronic Equipment

${ }^{2}$ European Union

${ }^{3}$ Restriction of Hazardous Substance

${ }^{4}$ Eco-Design Requirements for Energy Using Products
} 
promote the sustainable development efforts. Besides, reusing old and discarded items are economically attractive compared with their disposals. A review of closed loop supply chains (CLSC) is provided in Govindan and Soleimani [2]. Also some works in the literature, has emphasized the importance of inventory management in reverse supply chain (e.g. [3, 4]).

Although CLSC is comparatively a new term, the earliest reported work in this area was made by Schrady [5]. He proposed an inventory model for instantaneous producing and reproducing processes by considering infinite production rate and ignoring discarding cost. Nahmias and Rivera [6] extended the study of Schrady [5] by considering a finite storage and repair rate. Mabini et al. [7] developed the paper of Nahmias and Rivera [6] by considering a demand-dependent return rate and stock out service level constraint in a multi-product model. Koh et al. [8] extended the research of Nahmias and Rivera [6] by considering a finite capacity for repair. Richter [9] developed the work of Schrady [5] by extending an EOQ production, reproduction, and waste disposal model. Also Richter [10] indicated that the best recovery policy is a pure (bang-bang) policy in which all returns are either repaired or disposed; he further developed his work in later papers [10-13]. Similar to Richter [13], Teunter [14, 15], was one of the first authors in extending inventory models in reverse logistics. Teunter [16] has proposed a model in which all products cannot be recycled. Also he developed schrady's work by assuming several production and remanufacture cycles. Dobos and Richter [17] suggested a non-instantaneous producing and reproducing model in a single-cycle repair and production system. They also extended their earlier model [17] by assuming multiple products. Also Dobos and Richter [13] developed their earlier works by proposing a CLSC model in which all used items which are returned are not appropriate for remanufacturing. Along the line of researchers mentioned, other researchers have developed models with different assumptions such as, switching costs [18], entropy cost [19], backordered costs [20], learning during production and investment in learning [21, 22], disassembly of used items [23], considering the effect of energy, shipment, and waste scrapping costs simultaneously [24], and considering a situation in which a used product can be recycled for only a finite number of times [25].

Our research relates to three streams of literature: (1) considering manufactured and remanufactured products as different items, (2) the quality of returned items and return rate are variable, and (3) imperfect production. The first stream focuses on two different quality standards of the remanufactured and produced items. The above surveyed works mentioned in earlier paragraphs, as many other papers, have assumed that reproduced items are similar to the newly produced ones. Generally, customers do not perceive newly produced items and remanufactured ones as being equivalent [26, 27]. This limitation was relaxed by Jaber and El Saadany [28] as they assumed that remanufactured items have a lower level of quality than newly produced ones. Konstantaras et al. [29] incorporated inspection and sorting of returned items into their production/remanufacturing model. They also assumed that some recovered items has lower quality than reproduced or new purchased ones, so they can be sold in a secondary market at a reduced price. Hasanov et al. [30] developed the study of Jaber and El Saadany [28] by considering that unsatisfied demands for serviceable products lead to a full or partial backorder. Singh and Saxena [31] extended a CLSC model by considering produced and reproduced items as two different products with different quality standards for decaying items. They also assumed that items which are not appropriate for remanufacturing, are salvaged instead of wasting.

The second stream concentrates on the quality of returned items and return rate. Among this stream of literature, the following papers are particularly related to ours. El Saadany and Jaber [32] developed the studies of Dobos and Richter $[12,17]$ by considering a variable return rate of second hand products which 
depends on the the minimum acceptable quality level and buyback price. Also the formulation of the collection rate of second hand products, in this paper, is inspired by the demand function of the Vörös's work [33]. Their results indicated that a bang-bang strategy is no longer the best policy and a mixed strategy (joint production and reproduction) is best policy rather than a bang-bang strategy. Hwang et al. [34] like El Saadany and Jaber [32] used a return rate of second hand product as a function depends on the quality level and buyback price. They also assumed the quality of recycled products is a random variable and only those returned items that qualify an acceptance quality level are collected from customers and remanufactured. Giri and Sharma [35] developed an inventory model of CLSC by considering random return of used product, stochastic market demand, and supply disruptions. Dutta and et al. [36] proposed a multi-period recovery framework to examine the optimal buy-back price which improves the return rate of used products by considering uncertain demand and capacity. Guo and Liang [37] developed a recycling and production model by examining the correlation of the minimum acceptable quality level of returns with reproduction and buyback cost. They also assumed that quality level of returns is uniformly distributed. .Aybek et al. [38] analyzed the effect of the quality of returns on reproduction process. Recently Guo and Ya [39] proposed a stochastic model that the collection rate, buy-back price, and reproducing cost depend on quality levels of returned items which follows an exponential distribution. Furthermore, as assumed by some researchers like Aybek et al. [38]; Galbreth [40]; Li \& Da [41] the distribution of the quality level of returns can have normal or Gamma P.D.F.s.

Another stream of the related literature studies a production and remanufacturing systems that can produce defectives. One common assumption which is considered in all of mentioned studies, is that the production process is perfect. However, in any production system, production of defective items is inevitable due to many reasons such machinery defects, transit in production process, and other related factors. Jamal et al. [42] investigated a deterministic model characterized by defective items to optimize the batch size. Cárdenas-Barrón et al. [43] proposed an improved algorithm for an EMQ model with rework to derive the optimal decisions of order quantity and number of shipments jointly. They also in a further work, obtained the optimal decisions of replenishment quantity and number of deliveries in an EMQ model characterized with multiple deliveries and rework process [44]. Sarkar et al. [45] extended an imperfect EPQ model by considering time-dependent (quadratic) demand. They also assumed that the imperfect items are reworked to make the product perfect. Wee et al. [46] analyzed the optimal solutions of an EMQ model with rework process and planned backorders. Sarkar and Moon [47] extended an imperfect production model to examine the relationship between lead times, reorder point, and quantity improvement by considering lead time-dependent backorder rate. Sarkar et al. [48] extended an imperfect EPQ model by considering backorders, random defective rate, and rework process in a single level manufacturing system. Trevi no-Garza et al. [49] developed two algorithms to optimize the joint decisions on replenishment quantity and number of deliveries in a vendor-buyer EPQ system which is characterized with the imperfect production. Inderfurth et al. [50] studied the lot-sizing decisions in a joint production and rework model with the consideration of reworking of defectives. Sarkar et al. [51] addressed a model of machine breakdown and its corrective and preventive repairs for an EPQ system. In a recent work, Sarkar and Saren [52] developed an imperfect EPQ model which change from in-control state to out-of-control one. They also incorporated inspection policy, Type I and Type II errors, and warranty policy to make their model more realistic. Recently Giri and Sharma [53] investigated a multilevel CLSC by considering imperfect production process and reworking of defectives. Like El Saadany and Jaber [32], they considered quality based return rate. Also some related research can be found in 
Taleizadeh et al. [55, 56, 57, 58, 59, 60, 61, 62, 63, 64, 65], Taleizadeh and Pentico [66], Mohammadi et al. [67].

Moreover a brief literature review is presented in Table (1).

Based on this discussion, there is no model that considers single item with two different quality standards, variable return rate which is dependent on an acceptance quality level of returns, stochastic quality level of returned items, production of defective items, and rework process simultaneously. Generally, this paper contributes to the literature of CLSC model in the following ways:

Table 1. A brief literature review

\begin{tabular}{|c|c|c|c|c|c|c|c|c|c|c|c|c|c|c|c|}
\hline \multirow[b]{2}{*}{ Author } & \multicolumn{2}{|c|}{$\begin{array}{c}\text { finite } \\
\text { rate }\end{array}$} & \multirow[b]{2}{*}{ 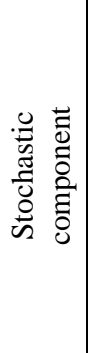 } & \multirow{2}{*}{ 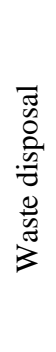 } & \multirow[b]{2}{*}{ 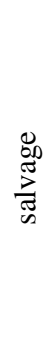 } & \multirow{2}{*}{ 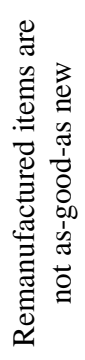 } & \multirow{2}{*}{ 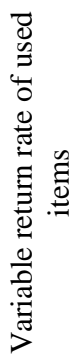 } & \multicolumn{3}{|c|}{ shortage } & \multirow{2}{*}{ 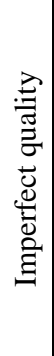 } & \multirow{2}{*}{ 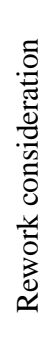 } & \multirow{2}{*}{ 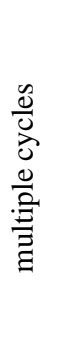 } & \multicolumn{2}{|c|}{ function } \\
\hline & 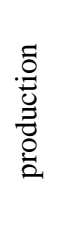 & 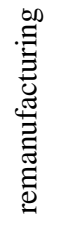 & & & & & & 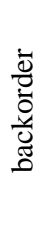 & 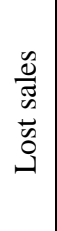 & 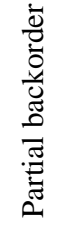 & & & &.$\Xi$ & 祃 \\
\hline Schrady, 1967 & & & & & & & & & & & & & & $\checkmark$ & \\
\hline Nahimas, 1979 & & $\checkmark$ & & & & & & & & & & & $\checkmark$ & $\checkmark$ & \\
\hline Richter, 1996a, $b, 1997$ & & & & $\checkmark$ & & & & & & & & & $\checkmark$ & $\checkmark$ & \\
\hline Teunter, $2001 \mathrm{~b}$ & & & & $\checkmark$ & & & & & & & & & $\checkmark$ & $\checkmark$ & \\
\hline Teunter, 2002 & & & $\sqrt{ }$ & $\checkmark$ & & & & & & & & & $\checkmark$ & $\checkmark$ & \\
\hline Koh et al., 2002 & & & & & & & & & & & & & $\checkmark$ & $\checkmark$ & \\
\hline $\begin{array}{c}\text { Dobos \& Richter, 1999, 2004, } \\
2006\end{array}$ & $\checkmark$ & $\checkmark$ & & $\checkmark$ & & & & & & & & & $\checkmark$ & $\checkmark$ & \\
\hline Konstantaras, 2006 & $\checkmark$ & $\checkmark$ & & & & & & $\checkmark$ & & & & & $\checkmark$ & $\checkmark$ & \\
\hline Konstantaras, 2010 & & & & & & $\checkmark$ & & & & & & & $\checkmark$ & $\checkmark$ & \\
\hline Jaber and El Saadany, 2009 & & & & & & $\checkmark$ & & & $\checkmark$ & & & & $\checkmark$ & $\checkmark$ & \\
\hline Hasanov et al., 2012 & & & & & & $\checkmark$ & & $\checkmark$ & & $\checkmark$ & & & $\checkmark$ & $\checkmark$ & \\
\hline Singh and Saxena, 2013 & & & & & $\checkmark$ & $\checkmark$ & & $\checkmark$ & & & & & & $\checkmark$ & \\
\hline El Saadany and Jaber, 2009 & $\checkmark$ & $\checkmark$ & & & & & $\checkmark$ & & & & & & $\checkmark$ & $\checkmark$ & \\
\hline Hwang et al., 2009 & $\checkmark$ & $\checkmark$ & $\checkmark$ & & & & $\checkmark$ & & & & & & & $\checkmark$ & \\
\hline Galbreth and Blackburn, 2006 & & & $\checkmark$ & $\checkmark$ & & & & & $\checkmark$ & & & & & $\checkmark$ & \\
\hline Guo and Liang, 2011 & & & $\checkmark$ & & & & $\checkmark$ & & & & & & $\checkmark$ & $\checkmark$ & \\
\hline Guo and Ya, 2014 & $\checkmark$ & $\checkmark$ & $\checkmark$ & & & & $\checkmark$ & & & & & & $\checkmark$ & $\checkmark$ & \\
\hline Jamal et al., 2004 & $\checkmark$ & $\checkmark$ & & & & & & & & & $\checkmark$ & & & $\checkmark$ & \\
\hline Inderfurth et al.,2005 & $\checkmark$ & $\checkmark$ & & & & & & & & & $\checkmark$ & $\checkmark$ & & $\checkmark$ & \\
\hline Giri and Sharma, 2015 & $\checkmark$ & $\checkmark$ & & & $\checkmark$ & & $\sqrt{ }$ & & & & $\checkmark$ & $\checkmark$ & $\checkmark$ & & $\checkmark$ \\
\hline This paper & $\checkmark$ & $\checkmark$ & $\checkmark$ & & $\checkmark$ & $\checkmark$ & $\checkmark$ & & $\checkmark$ & & $\checkmark$ & $\checkmark$ & $\checkmark$ & & $\checkmark$ \\
\hline
\end{tabular}

(1) We assume that produced and reproduced items have two different quality standards, so they are sold in the secondary market at a lower price. Since the demands for remanufactured and manufactured items are only fulfilled in their periods, this assumption leads to lost sales situations (Jaber and El Saadany [28]). Our research has a primary difference with the above study because we assume a CLSC model with a finite production and reproduction rate; however, they only considered infinite production and remanufacturing rate. Furthermore we consider the production cost, reproduction 
cost, inspection cost, purchasing cost of raw materials, purchasing cost of returned items, and salvage value while they did not consider these cost components.

(2) We assume the return rate of second hand item as a function of minimum acceptable quality level of returns $[32,39,53]$. Furthermore, in order to take the randomness of the quality level of returns into consideration, we propose three different probabilistic distribution functions for quality level of returns. Unlike Guo and Ya [39], we assume that the quality of used items is either triangularly or uniformly distributed. Also we assume that buyback cost, return rate, and reproduction cost are variable functions of quality level of returned products.

(3) We investigate a CLSC model by assuming that the returned items which are not suitable for remanufacturing are salvaged and sold in the tertiary market at a lesser price [31]. Also we assume that salvage value is influenced by the quality level of returns; however, many works in the literature assumed that returned items, which are not suitable for remanufacturing, are disposed.

(4) In the literature review, there are very few reverse logistics models considering defective items and rework process together. Unlike Giri and Sharma [53], this paper assumes that both production and remanufacturing process may produce a certain amount of defectives, and those defective items are reworked in the same production and remanufacturing set up.

In the following sections, Section 2, contains notations, assumptions, depiction and formulation of the systems. In Section 3 the solution procedures for the proposed models are presented. In Section 4 illustrative examples are presented, which clarify the solution procedures of the Section 3, and concluding remarks are presented in Section 5.

\section{Model formulation}

The conceptual figure of the proposed problem is depicted in Fig. 1 [28, 30]. However, Jaber and El Saadany [28] and Hasanov et al. [30] did not consider the tertiary market, random quality of returned items, and quality dependent return rate. We model a CLSC with two different quality standards. The demand for primary market is satisfied by newly manufactured items produced by raw materials purchased from outside. This type of products has a higher level of quality, but charges a higher price. Also the demand for secondary market is satisfied by remanufactured products produced by returned items purchased from customers. This type of products has a lower level of quality, but charges a lower price. The operations in presented reverse logistics can be classified into following activities: collecting the used items, inspection and sorting of the returned items and remanufacturing the items are suitable for remanufacturing. The collection rate of used products is considered as a function of the quality level announced by company. Manufacturer collects the used products from customers and brings them to a warehouse facility in which both inspection and sorting carry out. 


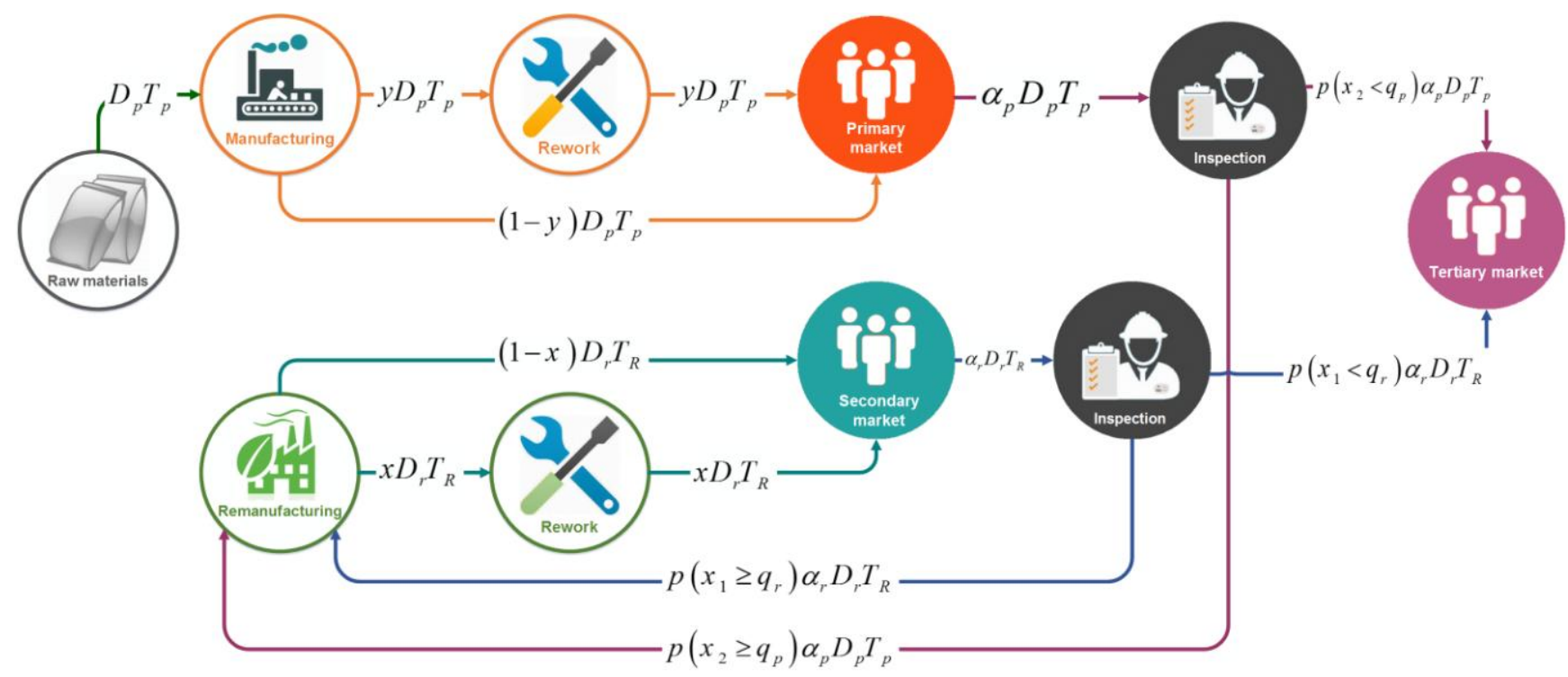

Manufactured products

Used products returned from primary market

Raw materials

Remanufactured products

Used products returned from secondary market

Fig. 1. Material flow for a CLSC system

As a returned product is inspected, then that is categorized as either remanufacturable or nonremanufacturable item. Actually returned items which do not satisfy the minimum quality level for remanufacturing, are salvaged and therefore sold at a lower price and are not collected again, while reproduced items are sold in a second market as the serviceable inventory.

Moreover the following assumptions are made:

1. Finite production and reproduction rates are assumed.

2. single type of item with two different qualities and prices is considered

3. The rates of demand for both new and second hand products are assumed to be certain and constant but different.

4. The manufacturing and remanufacturing processes are imperfect and a certain amount of manufactured items become defective and will be reworked in the same cycle. However, production of defectives at the time of reworking and remanufacturing is assumed to be negligible.

5. The quality of collected items is a random variable that follows a specific probability distribution function.

6. Planning horizon is infinite.

7. The minimum acceptable quality level of returns affects return rate of used products

8. Remanufacturing cost and salvage value is influenced by the quality of recycled items;

9. Shortage is permitted and unmet demands (manufactured or remanufactured) are lost sales.

10. Lead time is zero. 


\subsection{Modeling}

In this section the defined problem in section 2 is mathematically molded. In order to model the problem, the following notations are used throughout this paper.

\section{$\underline{\text { Parameters }}$}

$D_{r}$ : Rate of demand for reproduced product (unit/unit of time)

$D_{p}$ : Rate of demand for produced product (unit/unit of time)

$D_{r} / \gamma:$ Reproduction rate $(0<\gamma<1)$

$D_{p} / \beta$ : Production rate $(0<\beta<1)$

$Q_{r}$ : Reproduction lot size (in units) during $T$,

$Q_{s}:$ Salvage quantity (in units) during $T$

$Q_{p}:$ Reproduction lot size (in units) during $T$

$S_{r}$ : Remanufacturing setup cost per set up (\$/set up)

$S_{p}:$ Production setup cost (\$/set up)

$A$ : Fixed ordering cost of raw materials (\$/order)

$\alpha_{r}$ : Marginal buyback rate of items from the secondary market $\left(0<\alpha_{r}=b_{1} e^{-\varphi_{1} q_{r}}<1\right)$

$\alpha_{p}$ : Marginal buyback rate of newly produced items returned for recovery purposes $\left(0<\alpha_{p}=b_{2} e^{-\varphi_{2} q_{p}}<1\right)$

$a_{1} / \theta_{1}\left(a_{2} / \theta_{2}\right)$ : Parameter of the buyback cost function for returned items from the secondary (primary) market $\left(0<a_{1}, a_{2}<1\right)$

$b_{1} / \varphi_{1}\left(b_{2} / \varphi_{2}\right)$ : Parameter of the return rate function for used items returned from the secondary (primary) market $\left(0<b_{1}, b_{2}<1\right)$

$c_{1} / \delta_{1}\left(c_{2} / \delta_{2}\right)$ : Parameter of the reproduction cost function for returned items from the secondary (primary) market $\left(0<c_{1}, c_{2}<1\right)$

$R_{r}$ : Return rate for the items of the secondary market $\left(R_{r}=\alpha_{r} D_{r}\right)$

$R_{p}:$ Return rate for the items of the primary market $\left(R_{r}=\alpha_{r} D_{r}\right)$

$h_{r}$ : Carrying cost of reproduced product (\$/unit/unit of time)

$h_{p}$ : Carrying cost of new product (\$/unit/unit of time).

$h_{u}$ : Carrying cost of used item (\$/unit/unit of time)

$h_{\text {raw }}$ : Carrying cost of raw material (\$/unit/unit of time)

$h_{d f}$ : Carrying cost of defective item (\$/unit/unit of time)

$C_{\text {raw }}$ : unit raw materials cost (\$/unit)

$C_{b r}$ : Buyback cost ratio for the items of the secondary market (\$/unit)

$C_{b p}$ : Buyback cost ratio for the items of the primary market (\$/unit) 
$C_{r r}$ : Remanufacturing cost ratio for returned items from the secondary market (\$/unit)

$C_{p p}$ : Remanufacturing cost ratio for returned items from primary market (\$/unit)

$C_{p}$ : Manufacturing cost of $D_{p} T$ units (\$/unit)

$C_{l r}$ : Penalty cost of unmet demand for reproduced item (\$/unit)

$C_{l p}$ : Penalty cost of unmet demand for produced item (\$/unit)

$C_{I}:$ Unit inspection cost for the returned items (\$/unit)

$C_{R r}$ : Rework cost of remanufactured defective items (\$/unit)

$C_{R p}:$ Rework cost of produced defective items (\$/unit)

$x$ : Proportion of remanufactured defective items

$y$ : Proportion of produced defective items

$p_{r}$ : Unit selling price for remanufactured items (\$/unit)

$p_{p}:$ Unit selling price for produced items (\$/unit)

$p_{s r}$ : Selling price ratio for returned items from the secondary market which is not suitable for reproduction (\$/unit)

$p_{s p}$ : Selling price ratio for returned items from the primary market which is not suitable for reproduction (\$/unit)

$x_{1}$ : Quality level of cyclic returns from the secondary market (stochastic variable)

$x_{2}$ : Quality level of cyclic returns from the primary market (stochastic variable)

$e_{1}$ : Inferior limit of the quality probability distribution function (P.D.F.) function of returns from the secondary market

$e_{2}$ : Inferior limit of the quality P.D.F. function of returns from the primary market

$f_{1}$ : Superior limit of the quality P.D.F. function of returns from the secondary market

$f_{2}$ : Superior limit of the quality P.D.F. function of returns from the primary market

\section{Decision variables}

$T$ : The period length including production and reproduction cycles

$m:$ Number of reproduction cycles (integer variable)

$n:$ Number of production cycles (integer variable)

$q_{r}$ : Minimum acceptable quality level of returns from the secondary market $\left(0<q_{r}<1\right)$;

$q_{p}$ : Minimum acceptable quality level of returns from the primary market $\left(0<q_{p}<1\right)$;

As mentioned earlier, we assume that return rate, purchasing price of used items, and reproduction cost of used/returned items depend on the quality level of returned items. The return rate of used/returned items from the first and second markets, i.e., $R_{p}\left(q_{p}\right), R_{r}\left(q_{r}\right)$, respectively, are portions of the demand rate of first and second markets, i.e., $D_{p}, D_{r}$, respectively (see $\left.[39,53]\right)$. These quality factors explain the 
behavior of customers in returning of used items against the acceptance quality level which is determined by the manufacturer. In collecting process, if the quality level of a used item is too high, the collection rate will be very low and vice versa. The mathematical form of quality function of secondary and primary markets are assumed as $\alpha_{r}=b_{1} e^{-\varphi_{1} q_{r}}$ and $\alpha_{p}=b_{2} e^{-\varphi_{2} q_{p}}$, respectively, as shown in Fig. 2.

The remanufacturing costs of remanufacturable items, are portions of manufacturing cost, i.e., $C_{p}$. When the quality level of a returned product is many, the complexity of reproducing process is decreased and then reproduction cost is decreased and vice versa. The mathematical forms of remanufacturing cost ratios of secondary and primary markets are assumed to be $C_{r r}=c_{1} e^{\delta_{1}\left(1-x_{1}\right)}$ and $C_{r p}=c_{2} e^{\delta_{2}\left(1-x_{2}\right)}$, respectively, as shown in Fig. 3.

We define the buyback costs from the first and second markets as a fraction of the production cost of a new product (manufacturing cost plus raw material cost, $C_{r a w}+C_{p}$ ). Moreover as described in parameter definition section, $C_{b r}$ and $C_{b p}$, are defined as the ratios of buy back costs from the primary and secondary markets. Surely, if the quality level of returned items is good enough, the manufacturer will pay smaller remanufacturing cost and vice versa. On the other side, the buyback cost of the returned items with high quality level is more than those returned items with lower quality level as shown in Fig. 4. Adopting the negative exponential function from Vörös [33] and modifying it, the mathematical form of buyback cost ratios are assumed to be $C_{b r}=a_{1} e^{-\theta_{1}\left(1-x_{1}\right)}$ and $C_{b p}=a_{2} e^{-\theta_{2}\left(1-x_{2}\right)}$ as shown in Fig. 4.

Sales revenue of returned items from the first and second markets which are not suitable for remanufacturing and will be salvaged, is a fraction of $C_{r a w}+C_{p}$. The revenue from the salvages of returned items in the third market depends on the quality level of the items and increases exponentially when the quality increases. So, the mathematical form of sales revenue ratios in the second and first markets are assumed to be $p_{s r}=a_{1} e^{-\theta_{1}\left(1-x_{1}\right)}$ and $p_{s p}=a_{2} e^{-\theta_{2}\left(1-x_{2}\right)}$ respectively, as shown in Fig. 4 [40].

In this paper firstly we assume the quality level of returned items follows a general distribution function, then, the special cases of uniform and triangular distributions for the quality level of returned items are derived and analyzed.

The inventory of remanufactured items increases at the rate of $D_{r}\left(\frac{1}{\gamma}-1\right)$ and stops when the inventory level of $I_{r}$ is attained, and simultaneously the returned products level decreases at the rate of $p\left(x_{1} \geq q_{r}\right) R_{r}-\frac{D_{r}}{\gamma}$. After that, the defective items, remanufactured during the regular remanufacturing process, are reworked. Then the inventory of remanufactured items consumes at the rate of $D_{r}$. Similarly, the newly produced inventory builds up at the rate of $D_{p}\left(\frac{1}{\beta}-1\right)$ and then imperfect quality items produced during the regular production process are reworked. At the same time, raw material level reduces at the rate of $D_{p} / \beta$, inventory of returned products increases at a rate of $p\left(x_{2} \geq q_{p}\right) R_{p}$, and the inventory of newly produced items decreases at the rate of $D_{p}$. Fig. 5 illustrates the behavior of inventory of the serviceable, defective items, returned and raw material stocks. 


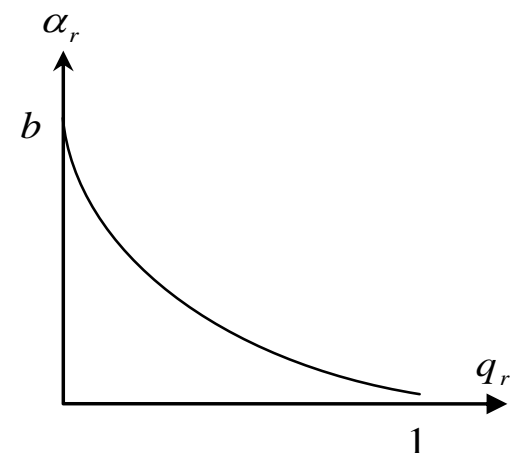

1

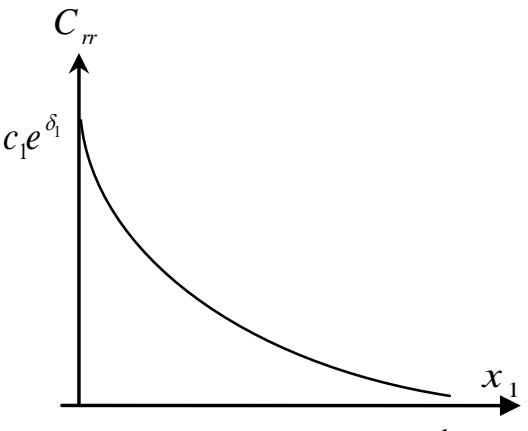

1

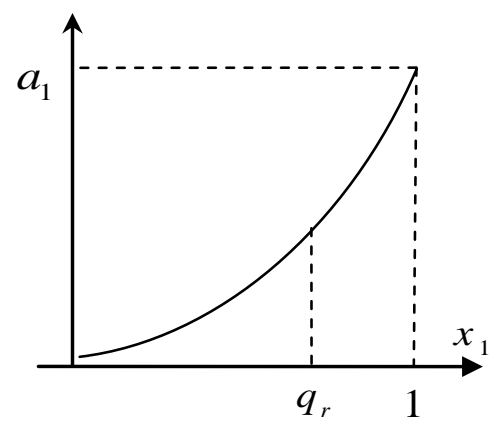

Fig. 2. Buyback cost ratio $p_{r}$

Fig. 3. Marginal buyback rate $\alpha_{r}$ Fig. 4. Remanufacturing cost ratio $C_{r r}$

In order to remanufacture the remanufacturable items, the manufacturer has to collect $p\left(x_{1} \geq q_{r}\right) \alpha_{r} D_{r} T_{R}$ repairable units from the second market over $T_{R}$ and $p\left(x_{2} \geq q_{p}\right) \alpha_{p} D_{p} T_{p}$ repairable units from the first market over $T_{p}$ (totally $p\left(x_{1} \geq q_{r}\right) \alpha_{r} D_{r} T_{R}+p\left(x_{2} \geq q_{p}\right) \alpha_{p} D_{p} T_{p}$ units).

Note that $D_{r} T_{R}$ is the remanufacturing lot size within $T$, so $D_{r} T_{R}=p\left(x_{1} \geq q_{r}\right) \alpha_{r} D_{r} T_{R}$ $+p\left(x_{2} \geq q_{p}\right) \alpha_{p} D_{p} T_{p}$ units. Accordingly,

$$
\frac{T_{p}}{T_{R}}=\frac{D_{r}\left(1-\alpha_{r} p\left(x_{1} \geq q_{r}\right)\right)}{D_{p}\left(\alpha_{p} p\left(x_{2} \geq q_{p}\right)\right)}
$$

In order to simplify the mathematical calculations, $T_{R}$ and $T_{P}$ can be re-written as follows:

$$
\begin{aligned}
& T_{p}=k T_{R}, k=\frac{D_{r}\left(1-\alpha_{r} p\left(x_{1} \geq q_{r}\right)\right)}{D_{p}\left(\alpha_{p} p\left(x_{2} \geq q_{p}\right)\right)} \\
& T_{R}+T_{p}=T \Rightarrow(1+k) T_{R}=T \Rightarrow T_{R}=\frac{1}{1+k} T \\
& \lambda=\frac{1}{1+k}, T_{R}=\lambda T, T_{R}=(1-\lambda) T
\end{aligned}
$$

The total expected cost per unit of time is comprised of the following parts. The set up and ordering costs are $\frac{S}{T}=\frac{m S_{r}+n S_{p}}{T}$ and $\frac{A}{T}$ respectively. The inventory holding cost expressions for raw materials (See Appendix B), newly produced, defective items, remanufactured, and returned items can be expressed as follows where $H_{r a w}, H_{p}, H_{d f}, H_{r}$ and $H_{u}$ denote them, respectively. 
Serviceable stock

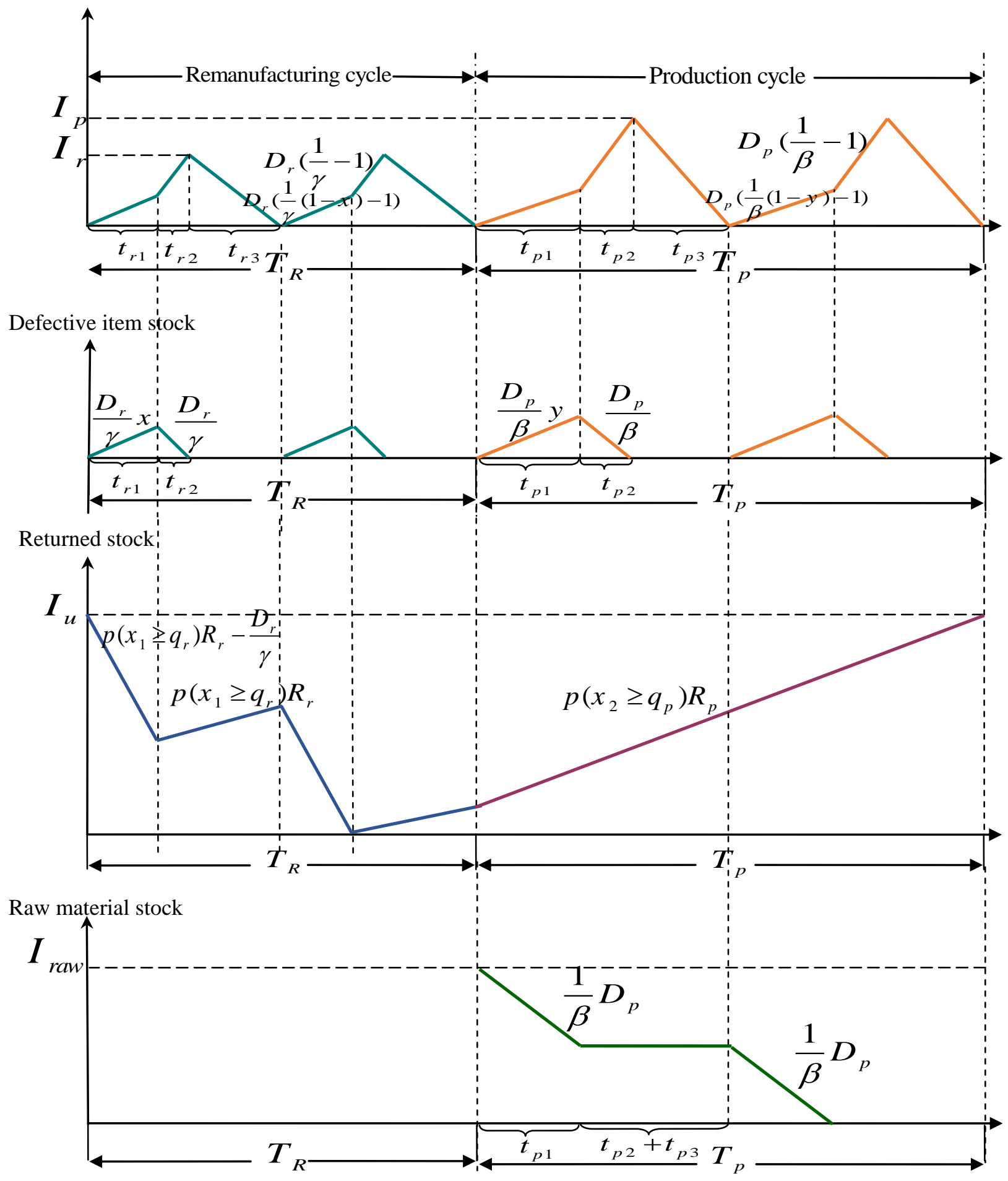

Manufactured products

Used products returned from primary market

Raw materials

Remanufactured products

Used products returned from secondary market

Fig. 5. Inventory status of the serviceable, defective, returned, and raw material stocks 


$$
\begin{gathered}
H_{r a w}=h_{r a w}\left(\overline{I_{r a w}}\right)=\frac{1}{2} h_{r a w} D_{p} T^{2} \frac{(1-\lambda)^{2}}{n}(\beta+n-1)=\frac{1}{2} D_{p} T^{2} \psi_{p}^{r a w} \\
H_{p}=h_{p}\left(\overline{I_{p}}\right)=\frac{1}{2} h_{p} D_{p} T^{2} \frac{(1-\lambda)^{2}}{n}\left(\beta\left(-y^{2}-y-1\right)+1\right)=\frac{1}{2} D_{p} T^{2} \psi_{p}^{p} \\
H_{r}=h_{r}\left(\overline{I_{r}}\right)=\frac{1}{2} h_{r} D_{r} T^{2} \frac{\lambda^{2}}{m}\left(\gamma\left(-x^{2}-x-1\right)+1\right)=\frac{1}{2} D_{r} T^{2} \psi_{r}^{r} \\
H_{d f}=h_{d f}\left(\overline{I_{d f}}\right)=\frac{1}{2} h_{d f}\left(D_{r} T^{2} \frac{\left(x+x^{2}\right) \gamma \lambda^{2}}{m}+D_{p} T^{2} \frac{\left(y+y^{2}\right) \beta(1-\lambda)^{2}}{n}\right)=\frac{1}{2} D_{r} T^{2} \psi_{r}^{d f}+\frac{1}{2} D_{p} T^{2} \psi_{p}^{d f} \\
H_{u}=h_{u}\left(\overline{I_{u}}\right)=\frac{1}{2} D_{r} h_{u} T^{2}\left[\frac{\gamma \lambda^{2}}{m}\left(1-\gamma \alpha_{r} p\left(x_{1} \geq q_{r}\right)\right)+\frac{(1-\gamma)^{2} \lambda^{2} \alpha_{r} p\left(x_{1} \geq q_{r}\right)}{m}\right. \\
\left.+\frac{(m-1) \lambda^{2}}{m}\left(1-\alpha_{r} p\left(x_{1} \geq q_{r}\right)\right)+2 \alpha_{r} p\left(x_{1} \geq q_{r}\right) \frac{(1-\gamma) \lambda(1-\lambda)}{m}\right] \\
+\frac{1}{2} D_{p} h_{u} T^{2}\left[\alpha_{p} p\left(x_{2} \geq q_{p}\right)(1-\lambda)^{2}\right]=\frac{1}{2} D_{r} T^{2} \psi_{r}^{u}+\frac{1}{2} D_{p} T^{2} \psi_{p}^{u}
\end{gathered}
$$

To simplify above formulas, let;

$$
\begin{aligned}
& \psi_{r}^{r}=h_{r} \frac{\lambda^{2}}{m}\left(\gamma\left(-x^{2}-x-1\right)+1\right) \\
& \psi_{p}^{p}=h_{p} \frac{(1-\lambda)^{2}}{n}\left(\beta\left(-y^{2}-y-1\right)+1\right) \\
& \psi_{r}^{d f}=h_{d f} \frac{\left(x+x^{2}\right) \gamma \lambda^{2}}{m} \\
& \psi_{p}^{d f}=h_{d f} \frac{\left(y+y^{2}\right) \beta(1-\lambda)^{2}}{n} \\
& \psi_{r}^{u}=h_{u}\left[\begin{array}{l}
\frac{\gamma \lambda^{2}}{m}\left(1-\gamma \alpha_{r} p\left(x_{1} \geq q_{r}\right)\right)+\frac{(1-\gamma)^{2} \lambda^{2} \alpha_{r} p\left(x_{1} \geq q_{r}\right)}{m} \\
+\frac{(m-1) \lambda^{2}}{m}\left(1-\alpha_{r} p\left(x_{1} \geq q_{r}\right)\right)+2 \alpha_{r} p\left(x_{1} \geq q_{r}\right) \frac{(1-\gamma) \lambda(1-\lambda)}{m}
\end{array}\right] \\
& \psi_{p}{ }^{u}=h_{u}\left[\alpha_{p} p\left(x_{2} \geq q_{p}\right)(1-\lambda)^{2}\right] \\
& \psi_{p}^{\text {raw }}=h_{\text {raw }}\left(\frac{(1-\lambda)^{2}(\beta+n-1)}{n}\right)
\end{aligned}
$$

For simplicity, we assume that:

$$
\begin{aligned}
& \psi_{p}=\psi_{p}^{p}+\psi_{p}^{u}+\psi_{p}^{r a w}+\psi_{p}^{d f} \\
& \psi_{r}=\psi_{r}^{r}+\psi_{r}^{u}+\psi_{r}^{d f}
\end{aligned}
$$

Derivations of Equations (5) to (9) are provided in Appendix A. Finally from Equations (5) to (9) the 
total holding cost can be obtained as follows

$$
H_{T, m, n}=\frac{H_{p}+H_{r}+H_{u}+H_{r a w}+H_{d f}}{T}=T D_{r} \frac{\psi_{r}}{2}+T D_{p} \frac{\psi_{p}}{2}
$$

Since we assumed that demands for newly produced items over $T_{R}$ and demands for reproduced items over $T_{p}$ are lost, the total lost sales cost within $T$ is the sum of $C_{r} D_{r} T_{p}$ and $C_{p} D_{p} T_{R}$. So the total shortage cost can be written as $\frac{1}{T}\left(C_{r l} D_{r} T_{p}+C_{p l} D_{p} T_{R}\right)=C_{l r} D_{r}(1-\lambda)+C_{l p} D_{p} \lambda$. Moreover the manufacturing costs per unit of time is $\frac{1}{T}\left(D_{p} T_{p}\right) C_{p}=\frac{1}{T}\left(D_{p}(1-\lambda) T\right) C_{p}$ and the remanufacturing costs per unit of time will be $\frac{1}{T}\left[p\left(x_{1} \geq q_{r}\right) \lambda T \alpha_{r} D_{r} E\left(C_{r r}\right) C_{p}\right]+\frac{1}{T} p\left[\left(x_{2} \geq q_{p}\right)(1-\lambda) T \alpha_{p} D_{p} E\left(C_{r p}\right) C_{p}\right]$ where $E\left(C_{r r}\right)=\int_{q_{r}}^{1} f\left(x_{1}\right) c_{1} e^{\delta_{1}\left(1-x_{1}\right)} d x_{1}$ and $E\left(C_{r p}\right)=\int_{q_{p}}^{1} f\left(x_{2}\right) c_{2} e^{\delta_{2}\left(1-x_{2}\right)} d x_{2}$.

Another part of the cost function is purchasing costs per unit time which consists of two elements. The first element is purchasing cost of raw materials, used for manufacturing of high quality items, which is equal to $\frac{1}{T}\left(\left(D_{p}(1-\lambda) T\right) C_{\text {raw }}\right)$ and the second element is purchasing cost of returned items, used for remanufacturing of low quality items, which is equal to $\frac{1}{T}\left[\lambda T \alpha_{r} D_{r} E\left(C_{b r}\right)\left(C_{r a w}+C_{p}\right)+(1-\lambda) T \alpha_{p} D_{p} E\left(C_{b p}\right)\left(C_{r a w}+C_{p}\right)\right]$ where $E\left(C_{b r}\right)=\int_{0}^{1} f\left(x_{1}\right) a_{1} e^{-\theta_{1}\left(1-x_{1}\right)} d x_{1}$ and $E\left(C_{b p}\right)=\int_{0}^{1} f\left(x_{2}\right) a_{2} e^{-\theta_{2}\left(1-x_{2}\right)} d x_{2}$.

Reworking cost per unit of time is $\frac{1}{T}\left(x D_{r} T_{R} C_{R r}+y D_{p} T_{p} C_{R p}\right)=\lambda x D_{r} C_{R r}+(1-\lambda) y D_{p} C_{R p}$ and the inspection cost per unit of time is $\frac{1}{T}\left(\alpha_{p} D_{p} T_{p}+\alpha_{r} D_{r} T_{r}\right) C_{I}=\frac{1}{T}\left(\alpha_{p} D_{p}(1-\lambda) T+\alpha_{r} D_{r} \lambda T\right) C_{I}$. Hence, the total expected cost per unit of time can be expressed as follows:

$$
\begin{aligned}
\operatorname{ETC}\left(T, m, n, q_{r}, q_{p}\right) & =\frac{S}{T}+\frac{A}{T}+\frac{T D_{r} \psi_{r}}{2}+\frac{T D_{p} \psi_{p}}{2}+C_{l r} D_{r}(1-\lambda)+C_{l p} D_{p} \lambda+\lambda x D_{r} C_{R r} \\
& +(1-\lambda) p\left(x_{2} \geq q_{p}\right) \alpha_{p} D_{p} E\left(C_{r p}\right) C_{p}+\lambda \alpha_{r} D_{r} E\left(C_{b r}\right)\left(C_{r a w}+C_{p}\right) \\
& +(1-\lambda) \alpha_{p} D_{p} E\left(C_{b p}\right)\left(C_{r a w}+C_{p}\right)+(1-\lambda) D_{p} C_{p}+\lambda p\left(x_{1} \geq q_{r}\right) \alpha_{r} D_{r} E\left(C_{r r}\right) C_{p} \\
& +(1-\lambda) y D_{p} C_{R p}+(1-\lambda) D_{p} C_{r a w}+\left(\alpha_{p} D_{p}(1-\lambda)+\alpha_{r} D_{r} \lambda\right) C_{I}
\end{aligned}
$$

Furthermore, the sales revenue per unit of time is comprised of three parts. The first is sales revenue from the produced items, i.e., $\frac{1}{T}\left(p_{p} D_{p} T_{p}\right)=(1-\lambda) p_{p} D_{p}$, the second part is the sale revenue from the remanufactured products, i.e., $\frac{1}{T}\left(p_{r} D_{r} T_{R}\right)=\lambda p_{r} D_{R}$, and the last portion is revenue of returned items are 
not suitable for remanufacturing which is equal to $\frac{1}{T}\left[p\left(x_{1} \leq q_{r}\right) \lambda T\left(\alpha_{r} D_{r}\right) E\left(p_{s r}\right)\left(C_{r a w}+C_{p}\right)\right]$ $+\frac{1}{T}\left[p\left(x_{2} \leq q_{p}\right)(1-\lambda) T\left(\alpha_{p} D_{p}\right) E\left(p_{s p}\right)\left(C_{r a w}+C_{p}\right)\right]$ where $\quad E\left(p_{s r}\right)=\int_{0}^{q_{r}} f\left(x_{1}\right) a_{1} e^{-\theta_{1}\left(1-x_{1}\right)} d x_{1} \quad$ and $E\left(p_{s p}\right)=\int_{0}^{q_{p}} f\left(x_{2}\right) a_{2} e^{-\theta_{2}\left(1-x_{2}\right)} d x_{2}$. Finally considering total costs and total revenues, the total profit function can be shown as below:

$$
\begin{aligned}
\operatorname{ETP}(T, & \left.m, n, q_{r}, q_{p}\right)=\left\{\begin{array}{c}
\text { sales revenue }- \text { setup costs - ordering cost - holding costs } \\
\text { - shortage cost }- \text { production cost }- \text { remanufacturing cost } \\
- \text { purchasing costs }- \text { reworking cost -inspection cost }
\end{array}\right\} \\
= & \lambda p_{r} D_{R}+(1-\lambda) p_{p} D_{p}+\lambda p\left(x_{1} \leq q_{r}\right)\left(\alpha_{r} D_{r}\right) E\left(p_{s r}\right)\left(C_{r a w}+C_{p}\right) \\
& +(1-\lambda) p\left(x_{2} \leq q_{p}\right)\left(\alpha_{p} D_{p}\right) E\left(p_{s p}\right)\left(C_{r a w}+C_{p}\right)-\frac{S}{T}-\frac{A}{T}-\frac{T D_{r} \psi_{r}}{2}-\frac{T D_{p} \psi_{p}}{2} \\
& -C_{l r} D_{r}(1-\lambda)-C_{l p} D_{p} \lambda-p\left(x_{1} \geq q_{r}\right) \lambda \alpha_{r} D_{r} E\left(C_{r r}\right) C_{p}-p\left(x_{2} \geq q_{p}\right)(1-\lambda) \alpha_{p} D_{p} E\left(C_{r p}\right) C_{p} \\
& -(1-\lambda) D_{p} C_{p}-\lambda x D_{r} C_{R r}-(1-\lambda) y D_{p} C_{R p}-\lambda \alpha_{r} D_{r} E\left(C_{b r}\right)\left(C_{r a w}+C_{p}\right) \\
& -(1-\lambda) \alpha_{p} D_{p} E\left(C_{b p}\right)\left(C_{r a w}+C_{p}\right)-(1-\lambda) D_{p} C_{r a w}-\left(\alpha_{p} D_{p}(1-\lambda)+\alpha_{r} D_{r} \lambda\right) C_{I}
\end{aligned}
$$

Theorem 1. The expected profit function shown in Equation (21) is a concave function over $T$.

Proof. See appendix C.

Since the profit function shown in Equation (21) is a concave function over $T$, setting the first derivative of Equation (21) equal to zero and solving it with respect to $T$, yields:

$$
T^{*}=\sqrt{\frac{2(S+A)}{D_{r} \psi_{r}+D_{p} \psi_{p}}}
$$

Where $\psi_{p}$ and $\psi_{r}$ are given in Equations (17) and (18). Substituting $T^{*}$ for $T$ in Equation (21), the total expected profit can be expressed as follows:

$$
\begin{aligned}
\operatorname{ETP}(m, & \left., q_{r}, q_{p}\right)=\lambda p_{r} D_{R}+(1-\lambda) p_{p} D_{p}+\lambda p\left(x_{1} \leq q_{r}\right)\left(\alpha_{r} D_{r}\right) E\left(p_{s r}\right)\left(C_{r a w}+C_{p}\right) \\
& +(1-\lambda) p\left(x_{2} \leq q_{p}\right)\left(\alpha_{p} D_{p}\right) E\left(p_{s p}\right)\left(C_{r a w}+C_{p}\right)-\sqrt{2(S+A)\left(D_{r} \psi_{r}+D_{p} \psi_{p}\right)} \\
& -C_{l r} D_{r}(1-\lambda)-C_{l p} D_{p} \lambda-p\left(x_{1} \geq q_{r}\right) \lambda \alpha_{r} D_{r} E\left(C_{r r}\right) C_{p}-p\left(x_{2} \geq q_{p}\right)(1-\lambda) \alpha_{p} D_{p} E\left(C_{r p}\right) C_{p} \\
& -(1-\lambda) D_{p} C_{p}-\lambda x D_{r} C_{R r}-(1-\lambda) y D_{p} C_{R p}-\lambda \alpha_{r} D_{r} E\left(C_{b r}\right)\left(C_{r a w}+C_{p}\right) \\
& -(1-\lambda) \alpha_{p} D_{p} E\left(C_{b p}\right)\left(C_{r a w}+C_{p}\right)-(1-\lambda) D_{p} C_{r a w}-\left(\alpha_{p} D_{p}(1-\lambda)+\alpha_{r} D_{r} \lambda\right) C_{I}
\end{aligned}
$$

The mathematical programming problem can be rewritten from Equation (23) as follows:

$$
Z=\text { Maximize } \operatorname{ETP}\left(m, n, q_{r}, q_{p}\right)
$$

\section{Subject to}


$0 \leq p\left(x_{1} \geq q_{r}\right) \leq 1$

$p_{\min } \leq p\left(x_{2} \geq q_{p}\right) \leq 1$

$e_{1} \leq q_{r} \leq f_{1}$

$e_{2} \leq q_{p} \leq f_{2}$

$n, m \geq 1$, are integers

Here we proposed the reasonable limitations for constraints (24-2) and (24-3). Consider case (a), $p\left(x_{1} \geq q_{r}\right)=0$ and $p\left(x_{2} \geq q_{p}\right)>0$, where manufacturer collects returned products only from the primary market at the rate of $p\left(x_{2} \geq q_{p}\right) \alpha_{p}$. Now consider case (b), $p\left(x_{1} \geq q_{r}\right)=0$ and $p\left(x_{2} \geq q_{p}\right)>0$, where manufacturer collects returned products only from the secondary market at the rate of $p\left(x_{1} \geq q_{r}\right) \alpha_{r}$. Case (b) is impossible because the reverse flow of returned items will be a closed loop of $D_{r}$ that has a limited and descending supply capacity that one time will be equal to zero, so collected items from the primary market must feed the secondary market. Consequently, $p_{\min }$ can never be equal to zero and $p\left(x_{2} \geq q_{p}\right) \geq p_{\min }$. Furthermore, in a lot of countries, governmental laws compel firms to establish recycling programs such as recycling, remanufacturing, and refurbishing (e.g., $[3,54])$. Therefore, it is assumed that some collections must occur i.e., $p_{\min }>0$.

\subsubsection{Case A: The quality of returned items follows a uniform distribution}

In this case, it is assumed that the qualities of returns from the primary and secondary markets follow non-identical independent uniform P.D.F.s within $\left[e_{1}, f_{1}\right]$ and $\left[e_{2}, f_{2}\right]$, respectively. Where parameters $e_{1}$ and $e_{2}$ are the inferior limits, and parameters $f_{1}$ and $f_{2}$ are the superior limits of the uniform P.D.F.s As both limits represent the quality level of returned items, obviously they must satisfy constraints: $0<e_{1}<f_{1}<1$ and $0<e_{2}<f_{2}<1$. In this case the probability density functions of the quality of returned items are:

$X_{1}\left(x_{1}\right)=\left\{\begin{array}{lc}\frac{1}{f_{1}-e_{1}} & 0<e_{1} \leq \mathrm{x}_{1} \leq f_{1}<1 \\ 0 & \text { others }\end{array}\right.$
$X_{2}\left(x_{2}\right)=\left\{\begin{array}{lc}\frac{1}{f_{2}-e_{2}} & 0<e_{2} \leq \mathrm{x}_{2} \leq f_{2}<1 \\ 0 & \text { others }\end{array}\right.$

By considering $x_{1}$ and $x_{2}$ as a stochastic variable following uniform P.D.F.s,$p\left(x_{1} \geq q_{r}\right)$, $p\left(x_{2} \geq q_{p}\right), \quad p\left(x_{1} \leq q_{r}\right), \quad p\left(x_{2} \leq q_{p}\right), \quad E\left(C_{r r}\right), \quad E\left(C_{r p}\right), \quad E\left(C_{b r}\right), \quad E\left(C_{b p}\right), \quad E\left(p_{s r}\right)$, and 
$E\left(p_{s p}\right)$ can be calculated as presented in Appendix E. Therefore, by substituting these relations in Equation (23), the total expected profit function can be written as following:

$$
\begin{aligned}
& E_{P_{u}}\left(m, n, q_{r}, q_{p}\right)=\lambda p_{r} D_{R}+(1-\lambda) p_{p} D_{p}+\lambda\left(\frac{q_{r}-e_{1}}{f_{1}-e_{1}}\right)\left(\alpha_{r} D_{r}\right)\left(\frac{a_{1} e^{-\theta_{1}}}{\theta_{1}\left(f_{1}-e_{1}\right)}\left[e^{\theta_{1} q_{r}}-e^{\theta_{1} e_{1}}\right]\right) \\
& \quad \times\left(C_{r a w}+C_{p}\right)+(1-\lambda)\left(\frac{q_{p}-e_{2}}{f_{2}-e_{2}}\right)\left(\alpha_{p} D_{p}\right)\left(\frac{a_{2} e^{-\theta_{2}}}{\theta_{2}\left(f_{2}-e_{2}\right)}\left[e^{\theta_{2} q_{p}}-e^{\theta_{2} e_{2}}\right]\right)\left(C_{r a w}+C_{p}\right)-\sqrt{2(S+A)\left(D_{r} \psi_{r}+D_{p} \psi_{p}\right)} \\
& \quad-C_{l r} D_{r}(1-\lambda)-C_{l p} D_{p} \lambda-\lambda\left(\frac{f_{1}-q_{r}}{f_{1}-e_{1}}\right) \alpha_{r} D_{r}\left(\frac{c_{1} e^{\delta_{1}}}{\delta_{1}\left(f_{1}-e_{1}\right)}\left[e^{-\delta_{1} q_{r}}-e^{-\delta_{f} f_{1}}\right]\right) C_{p}-(1-\lambda)\left(\frac{f_{2}-q_{p}}{f_{2}-e_{2}}\right) \alpha_{p} D_{p} \\
& \quad \times\left(\frac{c_{2} e^{\delta_{2}}}{\delta_{2}\left(f_{2}-e_{2}\right)}\left[e^{-\delta_{2} q_{p}}-e^{-\delta_{2} f_{2}}\right]\right) C_{p}-\lambda \alpha_{r} D_{r}\left(\frac{a_{1} e^{-\theta_{1}}}{\theta_{1}\left(f_{1}-e_{1}\right)}\left[e^{\theta_{1} f_{1}}-e^{\theta_{1} e_{1}}\right]\right)\left(C_{r a w}+C_{p}\right)-(1-\lambda) \alpha_{p} D_{p} \\
& \quad \times\left(\frac{a_{2} e^{-\theta_{2}}}{\theta_{2}\left(f_{2}-e_{2}\right)}\left[e^{\theta_{2} f_{2}}-e^{\theta_{2} e_{2}}\right]\right)\left(C_{r a w}+C_{p}\right)-(1-\lambda) D_{p} C_{p}-\lambda x D_{r} C_{R r}-(1-\lambda) y D_{p} C_{R p}-(1-\lambda) D_{p} C_{r a w} \\
& \quad-\left(\alpha_{p} D_{p}(1-\lambda)+\alpha_{r} D_{r} \lambda\right) C_{I}
\end{aligned}
$$

So, by substituting Equations $p\left(x_{1} \geq q_{r}\right)$ and $p\left(x_{2} \geq q_{p}\right)$, which are provided in Appendix E, in Equations (24-2) and (24-3) respectively, after some algebra, the mathematical programming problem can be written as follows:

$Z=$ Maximize $\operatorname{ETP}_{u}\left(m, n, q_{r}, q_{p}\right)$

Subject to

$e_{1} \leq q_{r} \leq f_{1}$

$e_{2} \leq q_{p} \leq f_{2}-p_{\text {min }}\left(f_{2}-e_{2}\right)$

$n, m \geq 1$, are int egers

\subsubsection{Case B: The quality of returned items follows a triangular distribution}

In this case, we assume that the qualities of returned items from the primary and secondary markets follow non-identical independent simplified triangular PD.F. $\mathrm{s}$ with parameters $\left[e_{1}, g_{1}, f_{1}\right]$ and $\left[e_{2}, g_{2}, f_{2}\right]$ respectively, where parameters $e_{1}, e_{2}$ are the inferior limits, parameters $f_{1}, f_{2}$ are the superior limits, and $g_{1}, g_{2}$ are the mode of the triangular P.D.F.s. For simplicity, we assumed two sub-cases based on the value of $g_{1}, g_{2}$. In sub-case B.1, the P.D.F. of the quality level of returned items is an ascending function and in sub-case B.2 is a descending function.

Sub-case B.1: In sub-case B.1 we assumed that $g_{1}=f_{1}$ and $g_{2}=f_{2}$. The probability density functions of the quality of returned items are given by: 


$$
\begin{aligned}
& X_{1}\left(x_{1}\right)=\left\{\begin{array}{lc}
\frac{2\left(x_{1}-e_{1}\right)}{\left(f_{1}-e_{1}\right)^{2}} & 0 \leq e_{1} \leq \mathrm{x}_{1} \leq g_{1}=f_{1} \\
0 & \text { other }
\end{array}\right. \\
& X_{2}\left(x_{2}\right)=\left\{\begin{array}{lc}
\frac{2\left(x_{2}-e_{2}\right)}{\left(f_{2}-e_{2}\right)^{2}} & 0 \leq e_{2} \leq \mathrm{x}_{2} \leq g_{2}=f_{2} \\
0 & \text { other }
\end{array}\right.
\end{aligned}
$$

The probability density functions of the quality level of returned items when $g_{1}=f_{1}$ and $g_{2}=f_{2}$ is an ascending function as depicted in Appendix D. It means that in this sub-case, the return probability of high quality items is more than low ones. since all parameters represent the quality level of returned items, obviously they must satisfy constraints $0<e_{1} \leq g_{1}=f_{1}<1$ and $0<e_{2} \leq g_{1}=f_{2}<1$. To reduce the complexity of the optimization problem, $p\left(x_{1} \geq q_{r}\right), p\left(x_{2} \geq q_{p}\right), p\left(x_{1} \leq q_{r}\right), p\left(x_{2} \leq q_{p}\right), E\left(C_{r r}\right)$, $E\left(C_{p p}\right), E\left(C_{b r}\right), E\left(C_{b p}\right), E\left(p_{s r}\right)$, and $E\left(p_{s p}\right)$, can be calculated as provided in Appendix $\mathrm{F}$. Therefore, by substituting these relations in Equation (23), the total expected profit function can be written as following:

$$
\begin{aligned}
& \operatorname{ETP}_{t 1}\left(m, n, q_{r}, q_{p}\right)=\lambda p_{r} D_{R}+(1-\lambda) p_{p} D_{p} \\
& +\lambda\left(\frac{\left(q_{r}-e_{1}\right)^{2}}{\left(f_{1}-e_{1}\right)^{2}}\right)\left(\alpha_{r} D_{r}\right)\left(\begin{array}{l}
\frac{2 a_{1} e_{1}\left(e^{\theta_{1}\left(q_{r}-1\right)}-e^{\theta_{1}\left(e_{1}-1\right)}\right)}{\theta_{1}\left(f_{1}-e_{1}\right)^{2}} \\
-\frac{2 a_{1}\left(\left(e_{1} \theta_{1}-1\right) e^{\theta_{1}\left(e_{1}-1\right)}-\left(q_{r} \theta_{1}-1\right) e^{\theta_{1}\left(q_{r}-1\right)}\right)}{\theta_{1}^{2}\left(f_{1}-e_{1}\right)^{2}}
\end{array}\right)\left(C_{r a w}+C_{p}\right) \\
& +(1-\lambda)\left(\frac{\left(q_{p}-e_{2}\right)^{2}}{\left(f_{2}-e_{2}\right)^{2}}\right)\left(\alpha_{p} D_{p}\right)\left(\begin{array}{l}
\frac{2 a_{2} e_{2}\left(e^{\theta_{2}\left(q_{p}-1\right)}-e^{\theta_{2}\left(e_{2}-1\right)}\right)}{\theta_{2}\left(f_{2}-e_{2}\right)^{2}} \\
-\frac{2 a_{2}\left(\left(e_{2} \theta_{2}-1\right) e^{\theta_{2}\left(e_{2}-1\right)}-\left(q_{p} \theta_{2}-1\right) e^{\theta_{2}\left(q_{p}-1\right)}\right)}{\theta_{2}^{2}\left(f_{2}-e_{2}\right)^{2}}
\end{array}\right)\left(C_{\text {raw }}+C_{p}\right) \\
& -\sqrt{2(S+A)\left(D_{r} \psi_{r}+D_{p} \psi_{p}\right)}-C_{l r} D_{r}(1-\lambda)-C_{l p} D_{p} \lambda-\left(1-\frac{\left(q_{r}-e_{1}\right)^{2}}{\left(f_{1}-e_{1}\right)^{2}}\right) \lambda \alpha_{r} D_{r} \\
& \times\left(\frac{\left[2 c_{1}\left(\left(\delta_{1} q_{r}+1\right) e^{\delta_{1}\left(1-q_{r}\right)}-\left(\delta_{1} f_{1}+1\right) e^{\delta_{1}\left(1-f_{1}\right)}\right)\right]}{\delta_{1}^{2}\left(e_{1}-f_{1}\right)^{2}}+\frac{\left[2 c_{1} e_{1}\left(\frac{1}{e^{\delta_{1}\left(f_{1}-1\right)}}-\frac{1}{e^{\delta_{1}\left(q_{r}-1\right)}}\right)\right]}{\delta_{1}\left(e_{1}-f_{1}\right)^{2}}\right] C_{p} \\
& -\lambda \alpha_{r} D_{r}\left(\frac{2 a_{1} e_{1}\left(e^{\theta_{1}\left(e_{1}-1\right)}-e^{\theta_{1}\left(f_{1}-1\right)}\right)}{\theta_{1}\left(f_{1}-e_{1}\right)^{2}}-\frac{2 a_{1}\left(\left(e_{1} \theta_{1}-1\right) e^{\theta_{1}\left(e_{1}-1\right)}-\left(f_{1} \theta_{1}-1\right) e^{\theta_{1}\left(f_{1}-1\right)}\right)}{\theta_{1}^{2}\left(f_{1}-e_{1}\right)^{2}}\right)\left(C_{r a w}+C_{p}\right)
\end{aligned}
$$




$$
\begin{aligned}
& -\left(1-\frac{\left(q_{p}-e_{2}\right)^{2}}{\left(f_{2}-e_{2}\right)^{2}}\right)(1-\lambda) \alpha_{p} D_{p}\left(\begin{array}{l}
\frac{\left[2 c_{2}\left(\left(\delta_{2} q_{p}+1\right) e^{\delta_{2}\left(1-q_{p}\right)}-\left(\delta_{2} f_{2}+1\right) e^{\delta_{2}\left(1-f_{2}\right)}\right)\right]}{\delta_{2}^{2}\left(e_{2}-f_{2}\right)^{2}} \\
\left.+\frac{\left[2 c_{2} e_{2}\left(\frac{1}{e^{\delta_{2}\left(f_{2}-1\right)}}-\frac{1}{e^{\delta_{2}\left(q_{p}-1\right)}}\right)\right]}{\delta_{2}\left(e_{2}-f_{2}\right)^{2}}\right)
\end{array} C_{p}\right. \\
& -(1-\lambda) \alpha_{p} D_{p}\left(\frac{2 a_{2} e_{2}\left(e^{\theta_{2}\left(e_{2}-1\right)}-e^{\theta_{2}\left(f_{2}-1\right)}\right)}{\theta_{2}\left(f_{2}-e_{2}\right)^{2}}-\frac{2 a_{2}\left(\left(e_{2} \theta_{2}-1\right) e^{\theta_{2}\left(e_{2}-1\right)}-\left(f_{2} \theta_{2}-1\right) e^{\theta_{2}\left(f_{2}-1\right)}\right)}{\theta_{2}^{2}\left(f_{2}-e_{2}\right)^{2}}\right)\left(C_{r a w}+C_{p}\right) \\
& -(1-\lambda) D_{p} C_{p}-\lambda x D_{r} C_{R r}-(1-\lambda) y D_{p} C_{R p}-(1-\lambda) D_{p} C_{r a w}-\left(\alpha_{p} D_{p}(1-\lambda)+\alpha_{r} D_{r} \lambda\right) C_{I}
\end{aligned}
$$

By substituting Equations $p\left(x_{1} \geq q_{r}\right)$ and $p\left(x_{2} \geq q_{p}\right)$, which are provided in Appendix $\mathrm{F}$, in Equations (24-2) and (24-3) respectively, the mathematical programming problem can be written as follows:

$Z=$ Maximize $\operatorname{ETP}_{t 1}\left(m, n, q_{r}, q_{p}\right)$

\section{Subject to:}

$e_{1} \leq q_{r} \leq f_{1}$

$e_{2} \leq q_{p} \leq \min \left\{e_{2}+\sqrt{1-p_{\min }}\left(f_{2}-e_{2}\right), f_{2}\right\}$

$n, m \geq 1$, are integers

Sub-case B.2: In sub-case B.2 we assumed that $g_{1}=e_{1}$ and $g_{2}=e_{2}$. the P.D.F.s of the quality of returned items are given by:

$X_{1}\left(x_{1}\right)=\left\{\begin{array}{lc}\frac{2\left(f_{1}-x_{1}\right)}{\left(f_{1}-e_{1}\right)^{2}} & 0 \leq e_{1}=g_{1} \leq \mathrm{x}_{1} \leq f_{1} \\ 0 & \text { other }\end{array}\right.$
$X_{2}\left(x_{2}\right)= \begin{cases}\frac{2\left(f_{2}-x_{2}\right)}{\left(f_{2}-e_{2}\right)^{2}} & 0 \leq e_{2}=g_{2} \leq \mathrm{x}_{2} \leq f_{2} \\ 0 & \text { other }\end{cases}$

The P.D.F.s of the quality of returned items when $g_{1}=e_{1}$ and $g_{2}=e_{2}$, is a descending function as depicted in Appendix D. It means that in this sub-case the return probability of lower quality items is more than higher ones. To reduce the complexity of the optimization problem, $p\left(x_{1} \geq q_{r}\right), p\left(x_{2} \geq q_{p}\right)$, $p\left(x_{1} \leq q_{r}\right), p\left(x_{2} \leq q_{p}\right), E\left(C_{r r}\right), E\left(C_{r p}\right), E\left(C_{b r}\right), E\left(C_{b p}\right), E\left(p_{s r}\right)$, and $E\left(p_{s p}\right)$, can be obtained as shown in Appendix G. Therefore, by substituting these relations in Equation (23), the total expected profit function can be obtained as following: 


$$
\begin{aligned}
& \operatorname{ETP}_{t 2}\left(m, n, q_{r}, q_{p}\right)=\lambda p_{r} D_{R}+(1-\lambda) p_{p} D_{p} \\
& +\lambda\left(1-\frac{\left(f_{1}-q_{r}\right)^{2}}{\left(f_{1}-e_{1}\right)^{2}}\right)\left(\alpha_{r} D_{r}\right)\left(\begin{array}{l}
\frac{2 a_{1} f_{1}\left(e^{\theta_{1}\left(q_{r}-1\right)}-e^{\theta_{1}\left(e_{1}-1\right)}\right)}{\theta_{1}\left(f_{1}-e_{1}\right)^{2}} \\
+\frac{2 a_{1}\left(\left(e_{1} \theta_{1}-1\right) e^{\theta_{1}\left(e_{1}-1\right)}-\left(q_{r} \theta_{1}-1\right) e^{\theta_{1}\left(q_{r}-1\right)}\right)}{\theta_{1}^{2}\left(f_{1}-e_{1}\right)^{2}}
\end{array}\right)\left(C_{r a w}+C_{p}\right) \\
& +(1-\lambda)\left(1-\frac{\left(f_{2}-q_{p}\right)^{2}}{\left(f_{2}-e_{2}\right)^{2}}\right)\left(\alpha_{p} D_{p}\right)\left(\begin{array}{l}
\frac{2 a_{2} f_{2}\left(e^{\theta_{2}\left(q_{p}-1\right)}-e^{\theta_{2}\left(e_{2}-1\right)}\right)}{\theta_{2}\left(f_{2}-e_{2}\right)^{2}} \\
+\frac{2 a_{2}\left(\left(e_{2} \theta_{2}-1\right) e^{\theta_{2}\left(e_{2}-1\right)}-\left(q_{p} \theta_{2}-1\right) e^{\theta_{2}\left(q_{p}-1\right)}\right)}{\theta_{2}^{2}\left(f_{2}-e_{2}\right)^{2}}
\end{array}\right)\left(C_{r a w}+C_{p}\right) \\
& -\sqrt{2(S+A)\left(D_{r} \psi_{r}+D_{p} \psi_{p}\right)}-C_{l r} D_{r}(1-\lambda)-C_{l p} D_{p} \lambda-\left(\frac{\left(f_{1}-q_{r}\right)^{2}}{\left(f_{1}-e_{1}\right)^{2}}\right) \lambda \alpha_{r} D_{r} \\
& \times\left(\frac{\left[2 c_{1}\left(\left(\delta_{1} f_{1}+1\right) e^{\delta_{1}\left(1-f_{1}\right)}-\left(\delta_{1} q_{r}+1\right) e^{\delta_{1}\left(1-q_{r}\right)}\right)\right]}{\delta_{1}^{2}\left(e_{1}-f_{1}\right)^{2}}-\frac{\left[2 c_{1} f_{1}\left(\frac{1}{e^{\delta_{1}\left(f_{1}-1\right)}}-\frac{1}{e^{\delta_{1}\left(q_{r}-1\right)}}\right)\right]}{\delta_{1}\left(e_{1}-f_{1}\right)^{2}}\right) C_{p} \\
& -\left(\frac{\left(f_{2}-q_{p}\right)^{2}}{\left(f_{2}-e_{2}\right)^{2}}\right)(1-\lambda) \alpha_{p} D_{p}\left(\begin{array}{l}
\frac{\left[2 c_{2}\left(\left(\delta_{2} f_{2}+1\right) e^{\delta_{2}\left(1-f_{2}\right)}-\left(\delta_{2} q_{p}+1\right) e^{\delta_{2}\left(1-q_{p}\right)}\right)\right]}{\delta_{2}^{2}\left(e_{2}-f_{2}\right)^{2}} \\
-\frac{\left[2 c_{2} f_{2}\left(\frac{1}{e^{\delta_{2}\left(f_{2}-1\right)}}-\frac{1}{e^{\delta_{2}\left(q_{p}-1\right)}}\right)\right]}{\delta_{2}\left(e_{2}-f_{2}\right)^{2}}
\end{array}\right) C_{p} \\
& -\lambda \alpha_{r} D_{r}\left(\frac{2 a_{1} f_{1}\left(e^{\theta_{1}\left(f_{1}-1\right)}-e^{\theta_{1}\left(e_{1}-1\right)}\right)}{\theta_{1}\left(f_{1}-e_{1}\right)^{2}}+\frac{2 a_{1}\left(\left(e_{1} \theta_{1}-1\right) e^{\theta_{1}\left(e_{1}-1\right)}-\left(f_{1} \theta_{1}-1\right) e^{\theta_{1}\left(f_{1}-1\right)}\right)}{\theta_{1}^{2}\left(f_{1}-e_{1}\right)^{2}}\right)\left(C_{r a w}+C_{p}\right) \\
& -(1-\lambda) \alpha_{p} D_{p}\left(\frac{2 a_{2} f_{2}\left(e^{\theta_{2}\left(f_{2}-1\right)}-e^{\theta_{2}\left(e_{2}-1\right)}\right)}{\theta_{2}\left(f_{2}-e_{2}\right)^{2}}+\frac{2 a_{2}\left(\left(e_{2} \theta_{2}-1\right) e^{\theta_{2}\left(e_{2}-1\right)}-\left(f_{2} \theta_{2}-1\right) e^{\theta_{2}\left(f_{2}-1\right)}\right)}{\theta_{2}^{2}\left(f_{2}-e_{2}\right)^{2}}\right)\left(C_{r a w}+C_{p}\right) \\
& -(1-\lambda) D_{p} C_{p}-\lambda x D_{r} C_{R r}-(1-\lambda) y D_{p} C_{R p}-(1-\lambda) D_{p} C_{r a w}-\left(\alpha_{p} D_{p}(1-\lambda)+\alpha_{r} D_{r} \lambda\right) C_{I}
\end{aligned}
$$

By substituting Equations $p\left(x_{1} \geq q_{r}\right)$ and $p\left(x_{2} \geq q_{p}\right)$ in Equations (24-2) and (24-3), respectively and simplifying them, the mathematical programming problem can be written as follows:

$Z=$ Maximize $\operatorname{ETP}_{t 2}\left(m, n, q_{r}, q_{p}\right)$

Subject to

$$
\begin{aligned}
& e_{1} \leq q_{r} \leq f_{1} \\
& e_{2} \leq q_{p} \leq f_{2}-\sqrt{p_{\text {min }}}\left(f_{2}-e_{2}\right)
\end{aligned}
$$




\section{Solution procedure}

The aim of this section is to propose a solution method to determine the values of $m, n, q_{r}$ and $q_{p}$ that maximize the total expected profit $\operatorname{ETP}_{u}\left(m, n, q_{r}, q_{p}\right)$ in case A and the total expected profit $\operatorname{ETP}_{t 1}\left(m, n, q_{r}, q_{p}\right)$ or $\operatorname{ETP}_{t 2}\left(m, n, q_{r}, q_{p}\right)$ in case B with respect to their constraints. Note that this solution procedure is valid for both case A and case B. So, for simplicity, we use $\operatorname{ETP}\left(m, n, q_{r}, q_{p}\right)$ instead of $\operatorname{ETP}_{u}\left(m, n, q_{r}, q_{p}\right), \operatorname{ETP}_{t 1}\left(m, n, q_{r}, q_{p}\right)$, or $\operatorname{ETP}_{t 2}\left(m, n, q_{r}, q_{p}\right)$.

Step 1: For a set of $D_{r}, D_{p}, a_{1}, a_{2}, b_{1}, b_{2}, c_{1}, c_{2}, \varphi_{1}, \varphi_{2}, \theta_{1}, \theta_{2}, \delta_{1}, \delta_{2}, h_{r}, h_{p}, h_{u}, h_{\text {raw }}, \gamma$, $\beta, A, S_{r}, S_{p}, C_{p}, C_{n}, C_{I}, C_{r l}, C_{p l}, C_{R r}, C_{R p}, P_{r}, P_{p}, x$ and $y$, set $n=1$ and $m=1$, and then maximize $\operatorname{ETP}\left(m, n, q_{r}, q_{p}\right)$ using some computational software (e.g., Solver from Microsoft Excel, Mathematica, Matlab, etc.) with respect to its constraints. Record the total expected profit and their corresponding $q_{r}$ and $q_{p}$ values for the cases when $n=1, m=1$, where $q_{r}$ and $q_{p}$ are optimal for specific values of $n$ and $m$.

Step 2: Take $m=2,3,4, \ldots$ and repeat Step 1 to obtain the optimal value of $m$ and the corresponding optimal values of $q_{r}$ and $q_{p}$. When there is 1 production cycle, the optimal value of $m$, denoted by $m_{1}^{*}$, should satisfy the inequality $\operatorname{ETP}\left(m_{1}^{*}-1,1, q_{r}, q_{p}\right)<\operatorname{ETP}\left(m_{1}^{*}, 1, q_{r}, q_{p}\right)$ $>\operatorname{ETP}\left(m_{1}^{*}+1,1, q_{r}, q_{p}\right)$. Record the values of $\operatorname{ETP}^{*}\left(m_{1}^{*}, 1, q_{r}, q_{p}\right), q_{r}^{*}$ and $q_{p}^{*}$ for $n=1$.

Step 3: For the identical set of inputs in the first step, set $n=2$ and repeat Step 2. When there is 2 production cycles, the optimal value of $m$, denoted by $m_{2}^{*}$, and should satisfy inequality $\operatorname{ETP}\left(m_{2}^{*}-1,2, q_{r}, q_{p}\right)<\operatorname{ETP}\left(m_{2}^{*}, 2, q_{r}, q_{p}\right)>\operatorname{ETP}\left(m_{2}^{*}+1,2, q_{r}, q_{p}\right)$. Record the values of $\operatorname{ETP}^{*}\left(m_{2}^{*}, 2, q_{r}, q_{p}\right), q_{r}^{*}$ and $q_{p}^{*}$ for $n=2$.

Step 4: Compare $\operatorname{ETP}^{*}\left(m_{n}^{*}, n, q_{r}, q_{p}\right)$ for $n=1$ and $n=2$. If $\operatorname{ETP}\left(m_{1}^{*}, 1, q_{r}, q_{p}\right)>$ $\operatorname{ETP}\left(m_{2}^{*}, 2, q_{r}, q_{p}\right), \quad$ stop and $\operatorname{ETP}^{*}\left(m_{1}^{*}, 1, q_{r}, q_{p}\right)$ is the maximum profit. If $\operatorname{ETP}\left(m_{1}^{*}, 1, q_{r}, q_{p}\right)<\operatorname{ETP}\left(m_{2}^{*}, 2, q_{r}, q_{p}\right)$, then omit $\operatorname{ETP}^{*}\left(m_{1}^{*}, 1, q_{r}, q_{p}\right)$ and for $n=3$ repeat the first and the second steps. For $n=2$ and 3 compare $\operatorname{ETP}^{*}\left(m_{n}^{*}, n, q_{r}, q_{p}\right)$. If $\operatorname{ETP}\left(m_{2}^{*}, 2, q_{r}, q_{p}\right)>\operatorname{ETP}\left(m_{3}^{*}, 3, q_{r}, q_{p}\right)$, stop and $\operatorname{ETP}^{*}\left(m_{2}^{*}, 2, q_{r}, q_{p}\right)$ is the maximum profit. If $\operatorname{ETP}\left(m_{2}^{*}, 2, q_{r}, q_{p}\right)<\operatorname{ETP}\left(m_{3}^{*}, 3, q_{r}, q_{p}\right)$, then omit $\operatorname{ETP}^{*}\left(m_{2}^{*}, 2, q_{r}, q_{p}\right)$ and for $\mathrm{n}=4$ repeat steps 3 th and 4 th steps. 
Step 5: Repeat the first to the fifth steps and explore the optimal values by satisfying the following condition

$$
\operatorname{ETP}\left(m_{i-1}^{*}, i-1, q_{r}, q_{p}\right)<\operatorname{ETP}\left(m_{i}^{*}, i, q_{r}, q_{p}\right)>\operatorname{ETP}\left(m_{i+1}^{*}, i+1, q_{r}, q_{p}\right) \text {, for } \mathrm{i}=1,2,3,4, \text { etc. }
$$

After obtaining the optimal values for $T, m, n, q_{r}, q_{p}$, one can obtain $Q_{r}^{*}, Q_{p}^{*}$, and $Q_{s}^{*}$ as follows:

$Q_{r}^{*}=D_{r} T_{R}=D_{r} \lambda^{*} T^{*}$

$Q_{p}^{*}=D_{p} T_{p}=D_{p}\left(1-\lambda^{*}\right) T^{*}$

$Q_{s}^{*}=\alpha_{p}^{*} p^{*}\left(x_{2} \leq q_{p}\right) Q_{p}^{*}+\alpha_{r}^{*} p^{*}\left(x_{1} \leq q_{r}\right) Q_{r}^{*}$

Where $T_{R}^{*}, \lambda^{*}, \alpha_{r}^{*}, \alpha_{p}^{*}, p^{*}\left(x_{1} \leq q_{r}\right)$ and $p^{*}\left(x_{2} \leq q_{p}\right)$ are optimal values for $T_{R}, T_{p}, \lambda, \alpha_{r}, \alpha_{p}, p\left(x_{1} \leq q_{r}\right)$ and $p\left(x_{2} \leq q_{p}\right)$, respectively.

\section{Numerical and Experimental Computations}

Three numerical examples are provided in this section to illustrate the mathematical models and solution procedure presented in Section 2 and 3 numerically. We carry out numerical examples for three cases: (1) the quality of returned items is uniformly distributed, (2) the quality of returned items have an ascending triangular P.D.F., and (3) the quality of returned items have an descending P.D.F. We further conducted sensitivity analyses to study the effect of the main parameters on the model's behavior. The numerical values of the parameters in the production and remanufacturing system are considered as: $D_{r}=200, D_{p}=200, a_{1}=0.9, a_{2}=0.9, \theta_{1}=6, \theta_{2}=6, b_{1}=0.9, b_{2}=0.9, \varphi_{1}=2, \varphi_{2}=2, c_{1}=0.1$, $c_{2}=0.1, \delta_{1}=0.6, \delta_{2}=0.6, h_{r}=3, h_{p}=6, h_{u}=3, h_{\text {raw }}=3, h_{d f}=3, \gamma=0.5, \beta=0.2, S_{p}=144$, $S_{r}=72, A=50, C_{p}=15, C_{I}=3, C_{R r}=5, C_{R p}=6, C_{\text {Raw }}=50, C_{r l}=5, C_{p l}=6$, $p_{r}=150, p_{p}=200, x=0.07, y=0.05$.

\subsection{Example 1: (for case A)}

In this example, we assume that the quality of returned items from the first and second markets follow non-identical uniform P.D.F.s with parameters $\left[e_{1}, f_{1}\right]$ and $\left[e_{2}, f_{2}\right]$ respectively. The numerical values of the parameters of uniform P.D.F.s are considered as $e_{1}=0.05, e_{2}=0.1, f_{1}=0.9, f_{2}=0.95$. For this data set, using the solution procedure proposed in Section 3, the results shown in Table 1 can be obtained.

\subsection{Example 2: (for case B.1)}

As an illustration of the developed model for Case B.2, we assume that the quality of returned items from the first and second markets follow non-identical triangular P.D.F.s with parameters $\left[e_{1}, g_{1}, f_{1}\right]$ and $\left[e_{2}, g_{2}, f_{2}\right]$ respectively, where $g_{1}=f_{1}$ and $g_{2}=f_{2}$. The numerical values of the parameters of ascending triangular distribution are considered as $e_{1}=0.05, e_{1}=0.1, f_{1}=g_{1}=0.9, f_{2}=g_{2}=0.95$. 
Applying the solution procedure given in Section 3, we derived $m^{*}=1, n^{*}=1, q_{r}^{*}=0.05, q_{p}^{*}=0.94$ and $\operatorname{ETP}^{*}=25855.77$ are the optimal solutions.

Table 2: Numerical example1 related to case A illustrating the solution procedure

\begin{tabular}{ccccccc}
\hline Trial & $m$ & $n$ & $q_{r}$ & $q_{p}$ & $\operatorname{ETP}\left(m, n, q_{r}, q_{p}\right)$ & Notes \\
\hline 1 & 1 & 1 & 0.05 & 0.94 & 25093.47 & \\
2 & 1 & 2 & 0.05 & 0.94 & 25006.54 & $T P_{1,1}>T P_{1,2}$, therefore $m_{1}^{*}=1, T P_{1,1}=25093.47$ \\
3 & 2 & 1 & 0.05 & 0.94 & 25001.27 & \\
4 & 2 & 2 & 0.05 & 0.94 & 24936.35 & $T P_{2,1}>T P_{2,2}$, therefore $m_{2}^{*}=1, T P_{2,1}=25001.27$ \\
5 & & & & & & since $T P_{1,1}>T P_{2,1}, \mathrm{n}^{*}=1$, terminate \\
\hline
\end{tabular}

\subsection{Example 3: (for case B.2)}

In this example, we assume that the quality of returns from the first and second markets follow nonidentical triangular P.D.F.s with parameters $\left[e_{1}, g_{1}, f_{1}\right]$ and $\left[e_{2}, g_{2}, f_{2}\right]$ respectively, where $g_{1}=e_{1}$ and $g_{2}=e_{2}$. The numerical values of the parameters of ascending triangular P.D.F.s are considered as $e_{1}=g_{1}=0.05, e_{1}=g_{1}=0.1, f_{1}=0.9, f_{2}=0.95$. After solving the optimization model shown in Equation (35) using the solution procedure, the obtained results are $m^{*}=1, n^{*}=1, q_{r}^{*}=0.05$, $q_{p}^{*}=0.94$ and $E T P^{*}=24435.56$ as shown in Table (4). We compare numerical results of the three models in Table 5.

Table 3: Numerical example 2 related to case B.1 illustrating the solution procedure

\begin{tabular}{ccccccc}
\hline Trial & $m$ & $n$ & $q_{r}$ & $q_{p}$ & $\operatorname{ETP}\left(m, n, q_{r}, q_{p}\right)$ & Notes \\
\hline 1 & 1 & 1 & 0.05 & 0.94 & 25855.77 & \\
2 & 1 & 2 & 0.05 & 0.94 & 25768.84 & $T P_{1,1}>T P_{1,2}$, therefore $m_{1}^{*}=1, T P_{1,1}=25855.77$ \\
3 & 2 & 1 & 0.05 & 0.94 & 25763.57 & \\
4 & 2 & 2 & 0.05 & 0.94 & 25698.64 & $T P_{2,1}>T P_{2,2}$, therefore $m_{2}^{*}=1, T P_{1,1}=25763.57$ \\
5 & & & & & since $T P_{1,1}>T P_{2,1}, \mathrm{n}^{*}=1$, terminate
\end{tabular}

Table 4: numerical example 3 related to case B.2 illustrating the solution procedure.

\begin{tabular}{ccccccc}
\hline Trial & $m$ & $n$ & $q_{r}$ & $q_{p}$ & $\operatorname{ETP}\left(m, n, q_{r}, q_{p}\right)$ & Notes \\
\hline 1 & 1 & 1 & 0.05 & 0.94 & 24435.56 &
\end{tabular}




$\begin{array}{rrrrrrr}2 & 1 & 2 & 0.05 & 0.94 & 24347.55 & T P_{1,1}>T P_{1,2}, \text { therefore } m_{1}^{*}=1, T P_{1,1}=24435.56 \\ 3 & 2 & 1 & 0.05 & 0.94 & 24343.20 & \\ 4 & 2 & 2 & 0.05 & 0.94 & 24277.15 & T P_{2,1}>T P_{2,2}, \text { therefore } m_{2}^{*}=1, T P_{1,1}=24343.20 \\ 5 & & & & & & \text { since } T P_{1,1}>T P_{2,1}, \mathrm{n}^{*}=1, \text { terminate }\end{array}$

From the numerical experiments, we can conclude that the minimum cost is obtained for the case of B.1 which is a simplified triangular P.D.F. with ascending manner (See Table 5). The probability of return of items with higher quality in this case is more than cases A and B.2., so we conclude that when the probability of return of items with the high quality increases, the total expected profit will increase too.

Table 5: Comparing the results of three distribution functions

\begin{tabular}{ccccc}
\hline Case & $\operatorname{ETP}^{*}\left(m, n, q_{r}, q_{p}\right)$ & $Q_{r}^{*}$ & $Q_{s}^{*}$ & $Q_{p}^{*}$ \\
\hline & & & & \\
A & 25093.47 & 1.13 & 20.83 & 153.51 \\
B.1 & $\mathbf{2 5 8 5 5 . 7 7}$ & $\mathbf{1 . 1 2}$ & $\mathbf{2 0 . 5 9}$ & $\mathbf{1 5 3 . 5 1}$ \\
B.2 & 24435.56 & 0.00 & 21.12 & 153.83 \\
\hline
\end{tabular}

\section{Sensitivity analysis}

In this section, the sensitivity analysis is performed on the key parameters $D_{r}, D_{p}, p_{r}, p_{p}, C_{r a w}$, $C_{r l}, \varphi_{1}, \varphi_{2}, x$ and $y$ used in the proposed model, in order to study their effects on the remanufacturing policy and expected total profit. The results of the sensitivity analysis for the first case (Uniform PDF related to case $A$ ), the second case (triangular PDF related to case B.1) and also for the third case (triangular PDF related to case B.2) are shown in Tables (6) to (15) respectively.

Moreover the changes of production, remanufacturing and salvage batch size with respect to the changes of parameters in case A are shown in Fig. 6. It should be noted that cases B.1 and B.2 have almost identical behaviors as shown in Fig. 6; therefore, to avoid duplication, these figures are not presented here. Also the changes of expected total profit with respect to the parameters changes are shown in Fig. 7.

According to Tables (6) to (15), when the demand for remanufactured items increases, $Q_{r}^{*}$ and $E T P^{*}$ increase, and $Q_{s}^{*}, Q_{p}^{*}, q_{r}^{*}$, and $q_{p}^{*}$ decrease. In fact, the optimal solution gradually shifts to remanufacturing of used units collected from the both primary and secondary markets at the minimum acceptance quality level and maximum collection rate.

In order to gain more revenue, manufacturers are willing to remanufacture returned items and satisfy the demand for secondary market. Also in the matter of the environmental issue, the increasing return rate is favorable to both the CLSC and the environment because by increasing return rate, larger quantity of 
used products should be remanufactured or salvaged which can reduce the negative effect of disposing on environment. Therefore, increasing the demand for remanufactured items is beneficial for environmental issue.

When the demand for newly products increases, $Q_{p}^{*}, Q_{s}^{*}$ and $E T P^{*}$ increase, $Q_{r}^{*}$ decrease, and the optimal solution flips from remanufacturing all of collected items to remanufacturing used items collected from the secondary market. When the demand for primary market increases, manufacturer prefers to produce more new items that satisfy the demand for primary market, instead of using remanufactured items, because it can increase $E T P^{*}$. Also in the matter of the environmental impact, by increasing demand for newly produced items, return rate decreases and most of the used items are disposed by customers which has a negative effect on environment

When the purchasing cost of raw materials increases, $Q_{p}^{*}, Q_{s}^{*}$ and $E T P^{*}$ decrease, $Q_{r}^{*}$ increases and the optimal policy is collecting the used items from both markets instead of only the second market, so collection rate increases which reduces negative effect on environment. In fact, when the purchasing cost of raw materials increases, manufacturers prefer to remanufacture used products instead of producing new items are produced by raw materials.

Table 6: Sensitivity analysis of the optimal solutions for the parameter $D_{r}$

\begin{tabular}{|c|c|c|c|c|c|c|c|c|c|c|c|}
\hline \multirow{2}{*}{ 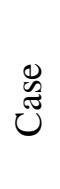 } & \multirow{2}{*}{ 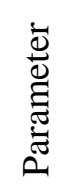 } & \multirow{2}{*}{ 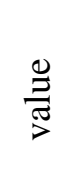 } & \multicolumn{8}{|c|}{ Value } & \multirow{2}{*}{$\begin{array}{c}\begin{array}{c}\text { Percentage } \\
\text { of changes }\end{array} \\
E T P^{*}\end{array}$} \\
\hline & & & $m^{*}$ & $n^{*}$ & $q_{r}^{*}$ & $q_{p}^{*}$ & $Q_{r}^{*}$ & $Q_{s}^{*}$ & $Q_{p}^{*}$ & $E T P^{*}$ & \\
\hline \multirow{6}{*}{$\varangle$} & \multirow{6}{*}{$D_{r}$} & 1 & 1 & 1 & 0.90 & 0.94 & 0.21 & 20.90 & 153.74 & 19330.71 & -22.97 \\
\hline & & 50 & 1 & 1 & 0.25 & 0.94 & 0.35 & 20.91 & 153.72 & 24672.55 & -1.68 \\
\hline & & 100 & 1 & 1 & 0.08 & 0.94 & 0.77 & 20.87 & 153.60 & 24673.61 & -1.67 \\
\hline & & 200 & 1 & 1 & 0.05 & 0.94 & 1.13 & 20.83 & 153.51 & 25093.47 & 0.00 \\
\hline & & 300 & 2 & 1 & 0.05 & 0.1 & 280.37 & 0.00 & 70.63 & 30243.69 & 20.52 \\
\hline & & 500 & 2 & 1 & 0.05 & 0.1 & 322.40 & 0.00 & 81.22 & 42737.90 & 70.31 \\
\hline \multirow{6}{*}{$\vec{\varphi}$} & \multirow{6}{*}{$D_{r}$} & 1 & 1 & 1 & 0.90 & 0.94 & 0.20 & 20.65 & 153.74 & 19250.18 & -25.55 \\
\hline & & 50 & 1 & 1 & 0.20 & 0.94 & 0.49 & 20.62 & 153.68 & 24642.69 & -4.69 \\
\hline & & 100 & 1 & 1 & 0.05 & 0.94 & 1.12 & 20.59 & 153.51 & 25117.83 & -2.85 \\
\hline & & 200 & 1 & 1 & 0.05 & 0.94 & 1.12 & 20.59 & 153.51 & 25855.77 & 0.00 \\
\hline & & 300 & 2 & 1 & 0.05 & 0.1 & 280.37 & 0.00 & 70.63 & 31846.11 & 23.17 \\
\hline & & 500 & 2 & 1 & 0.05 & 0.1 & 322.40 & 0.00 & 81.22 & 44995.77 & 74.03 \\
\hline \multirow{6}{*}{$\stackrel{\sim}{\varphi}$} & \multirow{6}{*}{$D_{r}$} & 1 & 1 & 1 & 0.77 & 0.94 & 0.00 & 21.12 & 153.83 & 25049.83 & 2.51 \\
\hline & & 50 & 1 & 1 & 0.05 & 0.94 & 0.00 & 21.12 & 153.83 & 24912.19 & 1.95 \\
\hline & & 100 & 1 & 1 & 0.05 & 0.94 & 0.00 & 21.12 & 153.83 & 24753.80 & 1.30 \\
\hline & & 200 & 1 & 1 & 0.05 & 0.94 & 0.00 & 21.12 & 153.83 & 24435.56 & 0.00 \\
\hline & & 300 & 2 & 1 & 0.1 & 0.05 & 228.96 & 10.27 & 91.24 & 28811.68 & 17.91 \\
\hline & & 500 & 2 & 1 & 0.1 & 0.05 & 322.40 & 19.12 & 81.22 & 40480.04 & 65.66 \\
\hline
\end{tabular}

Table 7: Sensitivity analysis of the optimal solutions for the parameter $D_{p}$ 


\begin{tabular}{|c|c|c|c|c|c|c|c|c|c|c|c|}
\hline \multirow{2}{*}{$\begin{array}{l}\ddot{E} \\
\tilde{E} \\
\tilde{E}\end{array}$} & \multirow{2}{*}{ 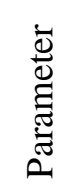 } & \multirow{2}{*}{$\stackrel{\stackrel{\varrho}{J}}{\stackrel{J}{J}}$} & \multicolumn{8}{|c|}{ Value } & \multirow{2}{*}{$\begin{array}{c}\begin{array}{c}\text { Percentage } \\
\text { of changes }\end{array} \\
E T P^{*}\end{array}$} \\
\hline & & & $m^{*}$ & $n^{*}$ & $q_{r}^{*}$ & $q_{p}^{*}$ & $Q_{r}^{*}$ & $Q_{s}^{*}$ & $Q_{p}^{*}$ & $E T P^{*}$ & \\
\hline \multirow{6}{*}{$\varangle$} & \multirow{6}{*}{$D_{p}$} & 1 & 1 & 1 & 0.05 & 0.1 & 0.08 & 1.47 & 10.85 & 273.12 & -98.91 \\
\hline & & 50 & 2 & 1 & 0.05 & 0.1 & 178.15 & 0.00 & 44.88 & 13572.08 & -45.91 \\
\hline & & 100 & 2 & 1 & 0.05 & 0.1 & 215.33 & 0.00 & 54.25 & 18409.19 & -26.64 \\
\hline & & 200 & 1 & 1 & 0.05 & 0.94 & 1.13 & 20.83 & 153.51 & 25093.47 & 0.00 \\
\hline & & 300 & 1 & 1 & 0.05 & 0.94 & 1.38 & 25.52 & 188.01 & 37589.49 & 49.80 \\
\hline & & 500 & 1 & 1 & 0.12 & 0.94 & 0.92 & 33.03 & 242.95 & 62552.90 & 149.28 \\
\hline \multirow{6}{*}{$\vec{\varphi}$} & \multirow{6}{*}{$D_{p}$} & 1 & 2 & 1 & 0.1 & 0.05 & 0.07 & 0.03 & 10.85 & 580.91 & -97.75 \\
\hline & & 50 & 2 & 1 & 0.1 & 0.05 & 178.15 & 0.58 & 44.88 & 14305.23 & -44.67 \\
\hline & & 100 & 2 & 1 & 0.1 & 0.05 & 215.33 & 0.70 & 54.25 & 19387.99 & -25.01 \\
\hline & & 200 & 1 & 1 & 0.05 & 0.94 & 1.12 & 20.59 & 153.51 & 25855.77 & 0.00 \\
\hline & & 300 & 1 & 1 & 0.05 & 0.94 & 1.37 & 25.22 & 188.01 & 38280.69 & 48.05 \\
\hline & & 500 & 1 & 1 & 0.07 & 0.94 & 1.44 & 32.57 & 242.81 & 62936.48 & 143.41 \\
\hline \multirow{6}{*}{$\stackrel{\sim}{\varphi}$} & \multirow{6}{*}{$D_{p}$} & 1 & 1 & 1 & 0.05 & 0.94 & 0.00 & 1.49 & 10.87 & -557.52 & -102.28 \\
\hline & & 50 & 2 & 1 & 0.1 & 0.05 & 178.15 & 10.56 & 44.88 & 12838.93 & -47.46 \\
\hline & & 100 & 2 & 1 & 0.1 & 0.05 & 215.33 & 12.77 & 54.25 & 17430.39 & -28.67 \\
\hline & & 200 & 1 & 1 & 0.05 & 0.94 & 0.00 & 21.12 & 153.83 & 24435.56 & 0.00 \\
\hline & & 300 & 1 & 1 & 0.05 & 0.94 & 0.00 & 25.87 & 188.40 & 37162.08 & 52.08 \\
\hline & & 500 & 1 & 1 & 0.05 & 0.94 & 0.00 & 33.40 & 243.22 & 62678.77 & 156.51 \\
\hline
\end{tabular}

Table 8: Sensitivity analysis of the optimal solutions for the parameter $C_{\text {raw }}$

\begin{tabular}{|c|c|c|c|c|c|c|c|c|c|c|c|}
\hline \multirow{2}{*}{$\begin{array}{l}\tilde{\Xi} \\
\tilde{U}\end{array}$} & \multirow{2}{*}{$\begin{array}{l}\dot{\bar{\Xi}} \\
\stackrel{0}{\Xi} \\
\bar{\Xi} \\
\tilde{\Xi}\end{array}$} & \multirow{2}{*}{ 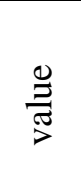 } & \multicolumn{8}{|c|}{ Value } & \multirow{2}{*}{$\begin{array}{c}\begin{array}{c}\text { Percentage } \\
\text { of changes }\end{array} \\
E T P^{*}\end{array}$} \\
\hline & & & $m^{*}$ & $n^{*}$ & $q_{r}^{*}$ & $q_{p}^{*}$ & $Q_{r}^{*}$ & $Q_{s}^{*}$ & $Q_{p}^{*}$ & $E T P^{*}$ & \\
\hline \multirow{5}{*}{$\ll$} & \multirow{5}{*}{$C_{r a w}$} & 40 & 1 & 1 & 0.05 & 0.94 & 1.13 & 20.83 & 153.51 & 26917.72 & 7.27 \\
\hline & & 60 & 2 & 1 & 0.05 & 0.94 & 1.27 & 23.50 & 173.18 & 23182.29 & -7.62 \\
\hline & & 80 & 2 & 1 & 0.11 & 0.13 & 186.72 & 11.82 & 93.86 & 20412.34 & -18.65 \\
\hline & & 100 & 2 & 1 & 0.05 & 0.1 & 245.46 & 0.00 & 61.84 & 19106.46 & -23.86 \\
\hline & & 120 & 2 & 1 & 0.05 & 0.1 & 245.46 & 0.00 & 61.84 & 17973.97 & -28.37 \\
\hline \multirow{5}{*}{$\bar{\varphi}$} & \multirow{5}{*}{$C_{\text {raw }}$} & 40 & 1 & 1 & 0.05 & 0.94 & 1.12 & 20.59 & 153.51 & 27573.41 & 6.64 \\
\hline & & 60 & 1 & 1 & 0.05 & 0.1 & 174.22 & 0.00 & 43.89 & 22397.82 & -13.37 \\
\hline & & 80 & 2 & 1 & 0.1 & 0.05 & 245.46 & 0.80 & 61.84 & 21110.07 & -18.35 \\
\hline & & 100 & 2 & 1 & 0.1 & 0.05 & 245.46 & 0.80 & 61.84 & 19774.48 & -23.52 \\
\hline & & 120 & 2 & 1 & 0.1 & 0.05 & 245.46 & 0.80 & 61.84 & 18438.88 & -28.69 \\
\hline \multirow{5}{*}{ ஸ் } & \multirow{5}{*}{$C_{r a w}$} & 40 & 1 & 1 & 0.05 & 0.94 & 0.00 & 21.12 & 153.83 & 26368.56 & 7.91 \\
\hline & & 60 & 1 & 1 & 0.23 & 0.26 & 65.89 & 37.07 & 125.47 & 22705.35 & -7.08 \\
\hline & & 80 & 1 & 1 & 0.15 & 0.17 & 108.75 & 26.12 & 99.49 & 20346.28 & -16.73 \\
\hline & & 100 & 2 & 1 & 0.09 & 0.11 & 193.99 & 14.91 & 89.35 & 18643.78 & -23.70 \\
\hline & & 120 & 2 & 1 & 0.05 & 0.1 & 245.46 & 0.00 & 61.84 & 17509.05 & -28.35 \\
\hline
\end{tabular}

Table 9: Sensitivity analysis of the optimal solutions for the parameter $C_{r l}$ 


\begin{tabular}{|c|c|c|c|c|c|c|c|c|c|c|c|}
\hline \multirow{2}{*}{ שै } & \multirow{2}{*}{ 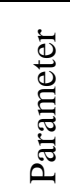 } & \multirow{2}{*}{$\frac{\mathscr{O}}{\overparen{J}}$} & \multicolumn{8}{|c|}{ Value } & \multirow{2}{*}{$\begin{array}{c}\text { Percentage } \\
\text { of changes } \\
\text { ETP* }\end{array}$} \\
\hline & & & $m^{*}$ & $n^{*}$ & $q_{r}^{*}$ & $q_{p}^{*}$ & $Q_{r}^{*}$ & $Q_{s}^{*}$ & $Q_{p}^{*}$ & $E T P^{*}$ & \\
\hline \multirow{5}{*}{$\varangle$} & \multirow{5}{*}{$C_{l r}$} & 3 & 1 & 1 & 0.05 & 0.94 & 1.13 & 20.83 & 153.51 & 25689.07 & 2.37 \\
\hline & & 6 & 1 & 1 & 0.05 & 0.94 & 1.13 & 20.83 & 153.51 & 25093.47 & 0.00 \\
\hline & & 9 & 1 & 1 & 0.05 & 0.94 & 1.13 & 20.83 & 153.51 & 24497.86 & -2.37 \\
\hline & & 12 & 1 & 1 & 0.05 & 0.94 & 1.13 & 20.83 & 153.51 & 23902.25 & -4.75 \\
\hline & & 15 & 1 & 1 & 0.28 & 0.27 & 81.17 & 23.57 & 117.05 & 22770.70 & -9.26 \\
\hline \multirow{5}{*}{$\vec{\varphi}$} & \multirow{5}{*}{$C_{l r}$} & 3 & 1 & 1 & 0.05 & 0.94 & 1.12 & 20.59 & 153.51 & 26451.41 & 2.30 \\
\hline & & 6 & 1 & 1 & 0.05 & 0.94 & 1.12 & 20.59 & 153.51 & 25855.77 & 0.00 \\
\hline & & 9 & 1 & 1 & 0.05 & 0.94 & 1.12 & 20.59 & 153.51 & 25260.12 & -2.30 \\
\hline & & 12 & 1 & 1 & 0.05 & 0.94 & 1.12 & 20.59 & 153.51 & 24664.48 & -4.61 \\
\hline & & 15 & 2 & 1 & 0.05 & 0.1 & 245.46 & 0.00 & 61.84 & 22751.23 & -12.01 \\
\hline \multirow{5}{*}{$\stackrel{N}{\oplus}$} & \multirow{5}{*}{$C_{l r}$} & 3 & 1 & 1 & 0.05 & 0.94 & 0.00 & 21.12 & 153.83 & 25035.55 & 2.46 \\
\hline & & 6 & 1 & 1 & 0.05 & 0.94 & 0.00 & 21.12 & 153.83 & 24435.56 & 0.00 \\
\hline & & 9 & 1 & 1 & 0.05 & 0.94 & 0.00 & 21.12 & 153.83 & 23835.57 & -2.46 \\
\hline & & 12 & 1 & 1 & 0.05 & 0.94 & 0.00 & 21.12 & 153.83 & 23235.58 & -4.91 \\
\hline & & 15 & 1 & 1 & 0.24 & 0.26 & 64.18 & 37.25 & 126.37 & 22884.62 & -6.35 \\
\hline
\end{tabular}

Table 10: Sensitivity analysis of the optimal solutions for the parameter $p_{r}$

\begin{tabular}{|c|c|c|c|c|c|c|c|c|c|c|c|}
\hline \multirow{2}{*}{$\begin{array}{l}\tilde{U} \\
\tilde{\tilde{z}} \\
\tilde{U}\end{array}$} & \multirow{2}{*}{ 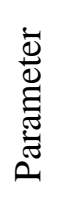 } & \multirow{2}{*}{$\frac{\mathscr{O}}{\stackrel{\pi}{J}}$} & \multicolumn{8}{|c|}{ Value } & \multirow{2}{*}{$\begin{array}{c}\begin{array}{c}\text { Percentage } \\
\text { of changes }\end{array} \\
E T P^{*}\end{array}$} \\
\hline & & & $m^{*}$ & $n^{*}$ & $q_{r}^{*}$ & $q_{p}^{*}$ & $Q_{r}^{*}$ & $Q_{s}^{*}$ & $Q_{p}^{*}$ & $E T P^{*}$ & \\
\hline \multirow{6}{*}{$\varangle$} & \multirow{6}{*}{$p_{r}$} & 130 & 1 & 1 & 0.05 & 0.94 & 1.13 & 20.83 & 153.51 & 25064.18 & -0.12 \\
\hline & & 150 & 1 & 1 & 0.05 & 0.94 & 1.13 & 20.83 & 153.51 & 25093.47 & 0.00 \\
\hline & & 156 & 1 & 1 & 0.05 & 0.94 & 1.13 & 20.83 & 153.51 & 25102.25 & 0.03 \\
\hline & & 157 & 1 & 1 & 0.27 & 0.3 & 74.10 & 24.13 & 121.08 & 24412.81 & -2.71 \\
\hline & & 170 & 1 & 1 & 0.16 & 0.18 & 116.77 & 15.43 & 93.94 & 25635.65 & 2.16 \\
\hline & & 190 & 2 & 1 & 0.1 & 0.06 & 234.37 & 7.60 & 68.01 & 28334.08 & 12.91 \\
\hline \multirow{6}{*}{$\vec{n}$} & \multirow{6}{*}{$p_{r}$} & 130 & 1 & 1 & 0.05 & 0.94 & 1.12 & 20.59 & 153.51 & 25826.73 & -0.11 \\
\hline & & 150 & 1 & 1 & 0.05 & 0.94 & 1.12 & 20.59 & 153.51 & 25855.77 & 0.00 \\
\hline & & 158 & 1 & 2 & 0.05 & 0.94 & 1.52 & 27.99 & 208.66 & 25775.19 & -0.31 \\
\hline & & 159 & 2 & 1 & 0.05 & 0.1 & 245.46 & 0.00 & 61.84 & 24551.23 & -5.05 \\
\hline & & 170 & 2 & 1 & 0.05 & 0.1 & 245.46 & 0.00 & 61.84 & 26308.49 & 1.75 \\
\hline & & 190 & 2 & 1 & 0.05 & 0.1 & 245.46 & 0.00 & 61.84 & 29503.53 & 14.11 \\
\hline \multirow{6}{*}{$\stackrel{\sim}{\varphi}$} & \multirow{6}{*}{$p_{r}$} & 130 & 1 & 1 & 0.05 & 0.94 & 0.00 & 21.12 & 153.83 & 24435.48 & 0.00 \\
\hline & & 150 & 1 & 1 & 0.05 & 0.94 & 0.00 & 21.12 & 153.83 & 24435.56 & 0.00 \\
\hline & & 157 & 1 & 1 & 0.05 & 0.94 & 0.00 & 21.12 & 153.83 & 24435.58 & 0.00 \\
\hline & & 158 & 1 & 1 & 0.25 & 0.27 & 61.86 & 38.11 & 127.58 & 24618.28 & 0.75 \\
\hline & & 170 & 1 & 1 & 0.16 & 0.18 & 52.66 & 23.09 & 131.01 & 25085.20 & 2.66 \\
\hline & & 190 & 2 & 1 & 0.1 & 0.06 & 129.44 & 4.41 & 84.34 & 27644.81 & 13.13 \\
\hline
\end{tabular}

Table 11: Sensitivity analysis of the optimal solutions for the parameter $p_{p}$ 


\begin{tabular}{|c|c|c|c|c|c|c|c|c|c|c|c|}
\hline \multirow{2}{*}{$\begin{array}{l}\mathbb{D} \\
\mathbb{ే} \\
\tilde{U}\end{array}$} & \multirow{2}{*}{ 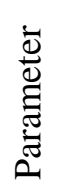 } & \multirow{2}{*}{ 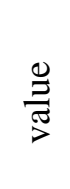 } & \multicolumn{8}{|c|}{ Value } & \multirow{2}{*}{$\begin{array}{c}\text { Percentage } \\
\text { of changes } \\
E T P^{*}\end{array}$} \\
\hline & & & $m^{*}$ & $n^{*}$ & $q_{r}^{*}$ & $q_{p}^{*}$ & $Q_{r}^{*}$ & $Q_{s}^{*}$ & $Q_{p}^{*}$ & $E T P^{*}$ & \\
\hline \multirow{6}{*}{$\varangle$} & \multirow{6}{*}{$p_{p}$} & 150 & 2 & 1 & 0.05 & 0.1 & 245.46 & 0.00 & 61.84 & 19925.27 & -20.60 \\
\hline & & 170 & 2 & 1 & 0.1 & 0.12 & 193.29 & 9.88 & 90.16 & 20871.17 & -16.83 \\
\hline & & 193 & 1 & 1 & 0.27 & 0.3 & 74.10 & 22.12 & 121.08 & 23012.81 & -8.29 \\
\hline & & 194 & 1 & 1 & 0.05 & 0.94 & 1.13 & 20.83 & 153.51 & 23902.25 & -4.75 \\
\hline & & 210 & 1 & 1 & 0.05 & 0.94 & 1.13 & 20.83 & 153.51 & 27078.82 & 7.91 \\
\hline & & 230 & 1 & 1 & 0.05 & 0.94 & 1.13 & 20.83 & 153.51 & 31049.54 & 23.74 \\
\hline \multirow{6}{*}{$\vec{\varphi}$} & \multirow{6}{*}{$p_{p}$} & 150 & 2 & 1 & 0.05 & 0.1 & 245.46 & 0.00 & 61.84 & 20980.30 & -18.86 \\
\hline & & 170 & 2 & 1 & 0.05 & 0.1 & 245.46 & 0.00 & 61.84 & 21785.27 & -15.74 \\
\hline & & 190 & 2 & 1 & 0.05 & 0.1 & 245.46 & 0.00 & 61.84 & 22590.23 & -12.63 \\
\hline & & 200 & 1 & 1 & 0.05 & 0.94 & 1.12 & 20.59 & 153.51 & 25260.12 & -2.30 \\
\hline & & 210 & 1 & 1 & 0.05 & 0.94 & 1.12 & 20.59 & 153.51 & 27245.60 & 5.38 \\
\hline & & 230 & 1 & 1 & 0.05 & 0.94 & 1.12 & 20.59 & 153.51 & 31216.56 & 20.73 \\
\hline \multirow{6}{*}{$\stackrel{\sim}{\varphi}$} & \multirow{6}{*}{$p_{p}$} & 150 & 2 & 1 & 0.05 & 0.1 & 195.80 & 0.00 & 88.33 & 18943.51 & -22.48 \\
\hline & & 170 & 2 & 1 & 0.1 & 0.12 & 109.80 & 12.49 & 98.76 & 20511.96 & -16.06 \\
\hline & & 190 & 1 & 1 & 0.24 & 0.26 & 66.49 & 37.54 & 125.15 & 22753.00 & -6.89 \\
\hline & & 200 & 1 & 1 & 0.05 & 0.94 & 0.00 & 21.12 & 153.83 & 24435.56 & 0.00 \\
\hline & & 210 & 1 & 1 & 0.05 & 0.94 & 0.00 & 21.12 & 153.83 & 26435.52 & 8.18 \\
\hline & & 230 & 1 & 1 & 0.05 & 0.94 & 0.00 & 21.12 & 153.83 & 30435.45 & 24.55 \\
\hline
\end{tabular}

Table 12: Sensitivity analysis of the optimal solutions for the parameter $\varphi_{1}$

\begin{tabular}{|c|c|c|c|c|c|c|c|c|c|c|c|}
\hline \multirow{2}{*}{$\begin{array}{l}\mathbb{E} \\
\tilde{U}\end{array}$} & \multirow{2}{*}{ 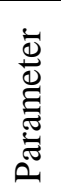 } & \multirow{2}{*}{ 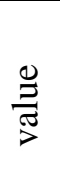 } & \multicolumn{8}{|c|}{ Value } & \multirow{2}{*}{$\begin{array}{c}\begin{array}{c}\text { Percentage } \\
\text { of changes }\end{array} \\
E T P^{*}\end{array}$} \\
\hline & & & $m^{*}$ & $n^{*}$ & $q_{r}^{*}$ & $q_{p}^{*}$ & $Q_{r}^{*}$ & $Q_{s}^{*}$ & $Q_{p}^{*}$ & $E T P^{*}$ & \\
\hline \multirow{5}{*}{$\ll$} & \multirow{5}{*}{$\varphi_{1}$} & 1 & 1 & 1 & 0.05 & 0.94 & 1.45 & 129.80 & 153.42 & 25156.63 & 0.25 \\
\hline & & 2 & 1 & 1 & 0.05 & 0.94 & 1.13 & 20.83 & 153.51 & 25093.47 & 0.00 \\
\hline & & 3 & 1 & 1 & 0.05 & 0.94 & 0.93 & 117.55 & 153.56 & 25030.56 & -0.25 \\
\hline & & 4 & 1 & 1 & 0.05 & 0.94 & 0.79 & 111.85 & 153.60 & 24969.31 & -0.49 \\
\hline & & 5 & 1 & 1 & 0.34 & 0.40 & 39.63 & 45.14 & 137.98 & 23952.22 & -4.55 \\
\hline \multirow{5}{*}{$\bar{\varphi}$} & \multirow{5}{*}{$\varphi_{1}$} & 1 & 1 & 1 & 0.05 & 0.94 & 1.44 & 128.27 & 153.42 & 25966.93 & 0.43 \\
\hline & & 2 & 1 & 1 & 0.05 & 0.94 & 1.12 & 122.09 & 153.51 & 25855.77 & 0.00 \\
\hline & & 3 & 1 & 1 & 0.05 & 0.94 & 0.92 & 116.17 & 153.56 & 25747.69 & -0.42 \\
\hline & & 4 & 1 & 1 & 0.05 & 0.94 & 0.79 & 110.53 & 153.60 & 25643.72 & -0.82 \\
\hline & & 5 & 1 & 1 & 0.42 & 0.50 & 38.46 & 27.43 & 138.47 & 23820.85 & -7.87 \\
\hline \multirow{5}{*}{$\stackrel{\sim}{n}$} & \multirow{5}{*}{$\varphi_{1}$} & 1 & 1 & 1 & 0.05 & 0.94 & 0.00 & 131.68 & 153.83 & 24464.38 & 0.12 \\
\hline & & 2 & 1 & 1 & 0.05 & 0.94 & 0.00 & 125.25 & 153.83 & 24435.56 & 0.00 \\
\hline & & 3 & 1 & 1 & 0.05 & 0.94 & 0.00 & 119.15 & 153.83 & 24408.14 & -0.11 \\
\hline & & 4 & 1 & 1 & 0.05 & 0.94 & 0.00 & 113.34 & 153.83 & 24382.05 & -0.22 \\
\hline & & 5 & 1 & 1 & 0.05 & 0.94 & 0.00 & 107.81 & 153.83 & 24357.23 & -0.32 \\
\hline
\end{tabular}

Table 13: Sensitivity analysis of the optimal solutions for the parameter $\varphi_{2}$ 


\begin{tabular}{|c|c|c|c|c|c|c|c|c|c|c|c|}
\hline \multirow{2}{*}{$\begin{array}{l}\mathscr{E} \\
\tilde{E} \\
\tilde{U}\end{array}$} & \multirow{2}{*}{ 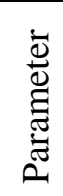 } & \multirow{2}{*}{$\frac{\mathscr{O}}{\stackrel{乛}{7}}$} & \multicolumn{8}{|c|}{ Value } & \multirow{2}{*}{$\begin{array}{c}\text { Percentage } \\
\text { of changes } \\
\text { ETP* }\end{array}$} \\
\hline & & & $m^{*}$ & $n^{*}$ & $q_{r}^{*}$ & $q_{p}^{*}$ & $Q_{r}^{*}$ & $Q_{s}^{*}$ & $Q_{p}^{*}$ & $E T P^{*}$ & \\
\hline \multirow{5}{*}{$\varangle$} & \multirow{5}{*}{$\varphi_{2}$} & 1 & 1 & 1 & 0.05 & 0.94 & 2.89 & 70.34 & 153.01 & 24559.21 & -2.13 \\
\hline & & 2 & 1 & 1 & 0.05 & 0.94 & 1.13 & 20.83 & 153.51 & 25093.47 & 0.00 \\
\hline & & 3 & 1 & 1 & 0.05 & 0.94 & 0.44 & 123.69 & 153.70 & 25305.17 & 0.84 \\
\hline & & 4 & 1 & 1 & 0.05 & 0.94 & 0.17 & 123.47 & 153.42 & 25372.53 & 1.11 \\
\hline & & 5 & 1 & 1 & 0.05 & 0.94 & 0.06 & 123.78 & 153.81 & 25420.74 & 1.30 \\
\hline \multirow{5}{*}{$\vec{\varphi}$} & \multirow{5}{*}{$\varphi_{2}$} & 1 & 1 & 1 & 0.42 & 0.46 & 78.16 & 16.99 & 118.12 & 23714.10 & -8.28 \\
\hline & & 2 & 1 & 1 & 0.05 & 0.94 & 1.12 & 58.25 & 153.51 & 25855.77 & 0.00 \\
\hline & & 3 & 1 & 1 & 0.05 & 0.94 & 0.43 & 122.24 & 153.70 & 26157.22 & 1.17 \\
\hline & & 4 & 1 & 1 & 0.05 & 0.94 & 0.16 & 122.30 & 153.78 & 26275.02 & 1.62 \\
\hline & & 5 & 1 & 1 & 0.05 & 0.94 & 0.06 & 122.33 & 153.81 & 26320.89 & 1.80 \\
\hline \multirow{5}{*}{$\stackrel{\sim}{\oplus}$} & \multirow{5}{*}{$\varphi_{2}$} & 1 & 1 & 1 & 0.05 & 0.94 & 0.00 & 79.07 & 153.82 & 24159.47 & -1.13 \\
\hline & & 2 & 1 & 1 & 0.05 & 0.94 & 0.00 & 125.25 & 153.83 & 24435.56 & 0.00 \\
\hline & & 3 & 1 & 1 & 0.05 & 0.94 & 0.00 & 125.25 & 153.83 & 24542.79 & 0.44 \\
\hline & & 4 & 1 & 1 & 0.05 & 0.94 & 0.00 & 125.25 & 153.83 & 24584.44 & 0.61 \\
\hline & & 5 & 1 & 1 & 0.05 & 0.94 & 0.00 & 125.25 & 153.83 & 24600.62 & 0.68 \\
\hline
\end{tabular}

Table 14: Sensitivity analysis of the optimal solutions for the parameter $x$

\begin{tabular}{|c|c|c|c|c|c|c|c|c|c|c|c|}
\hline \multirow{2}{*}{$\begin{array}{l}\mathscr{E} \\
\mathbb{E} \\
\tilde{U}\end{array}$} & \multirow{2}{*}{ 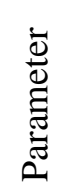 } & \multirow{2}{*}{$\stackrel{\cong}{\stackrel{\Xi}{\pi}}$} & \multicolumn{8}{|c|}{ Value } & \multirow{2}{*}{$\begin{array}{c}\begin{array}{c}\text { Percentage } \\
\text { of changes }\end{array} \\
\text { ETP }^{*}\end{array}$} \\
\hline & & & $m^{*}$ & $n^{*}$ & $q_{r}^{*}$ & $q_{p}^{*}$ & $Q_{r}^{*}$ & $Q_{s}^{*}$ & $Q_{p}^{*}$ & $E T P^{*}$ & \\
\hline \multirow{5}{*}{$\varangle$} & \multirow{5}{*}{$x$} & 0 & 1 & 1 & 0.05 & 0.94 & 1.13 & 20.83 & 153.51 & 25093.98 & 0.00 \\
\hline & & 0.05 & 1 & 1 & 0.05 & 0.94 & 1.13 & 20.83 & 153.51 & 25093.61 & 0.00 \\
\hline & & 0.1 & 1 & 1 & 0.05 & 0.94 & 1.13 & 20.83 & 153.51 & 25093.25 & 0.00 \\
\hline & & 0.2 & 1 & 1 & 0.05 & 0.94 & 1.13 & 20.83 & 153.51 & 25092.51 & 0.00 \\
\hline & & 0.5 & 1 & 1 & 0.05 & 0.94 & 1.13 & 20.83 & 153.51 & 25090.32 & -0.01 \\
\hline \multirow{5}{*}{$\ddot{n}$} & \multirow{5}{*}{$x$} & 0 & 1 & 1 & 0.05 & 0.94 & 1.12 & 58.25 & 153.51 & 25856.28 & 0.00 \\
\hline & & 0.05 & 1 & 1 & 0.05 & 0.94 & 1.12 & 58.25 & 153.51 & 25855.91 & 0.00 \\
\hline & & 0.1 & 1 & 1 & 0.05 & 0.94 & 1.12 & 58.25 & 153.51 & 25855.55 & 0.00 \\
\hline & & 0.2 & 1 & 1 & 0.05 & 0.94 & 1.12 & 58.25 & 153.51 & 25854.82 & 0.00 \\
\hline & & 0.5 & 1 & 1 & 0.05 & 0.94 & 1.12 & 58.25 & 153.51 & 25852.64 & -0.01 \\
\hline \multirow{5}{*}{ ๗ִ } & \multirow{5}{*}{$x$} & 0 & 1 & 1 & 0.05 & 0.94 & 0.00 & 125.25 & 153.83 & 24435.56 & 0.00 \\
\hline & & 0.05 & 1 & 1 & 0.05 & 0.94 & 0.00 & 125.25 & 153.83 & 24435.56 & 0.00 \\
\hline & & 0.1 & 1 & 1 & 0.05 & 0.94 & 0.00 & 125.25 & 153.83 & 24435.56 & 0.00 \\
\hline & & 0.2 & 1 & 1 & 0.05 & 0.94 & 0.00 & 125.25 & 153.83 & 24435.56 & 0.00 \\
\hline & & 0.5 & 1 & 1 & 0.05 & 0.94 & 0.00 & 125.25 & 153.83 & 24435.55 & 0.00 \\
\hline
\end{tabular}

Table 15: Sensitivity analysis of the optimal solutions for the parameter $y$ 


\begin{tabular}{|c|c|c|c|c|c|c|c|c|c|c|c|}
\hline \multirow{2}{*}{$\begin{array}{l}\mathscr{E} \\
\tilde{U} \\
\tilde{U}\end{array}$} & \multirow{2}{*}{ 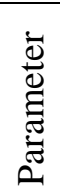 } & \multirow{2}{*}{ 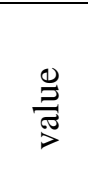 } & \multicolumn{8}{|c|}{ Value } & \multirow{2}{*}{$\begin{array}{c}\begin{array}{c}\text { Percentage } \\
\text { of changes }\end{array} \\
E T P^{*}\end{array}$} \\
\hline & & & $m^{*}$ & $n^{*}$ & $q_{r}^{*}$ & $q_{p}^{*}$ & $Q_{r}^{*}$ & $Q_{s}^{*}$ & $Q_{p}^{*}$ & $E T P^{*}$ & \\
\hline \multirow{5}{*}{$\varangle$} & \multirow{5}{*}{$y$} & 0 & 1 & 1 & 0.05 & 0.94 & 1.13 & 20.82 & 153.44 & 25152.74 & 0.24 \\
\hline & & 0.05 & 1 & 1 & 0.05 & 0.94 & 1.13 & 20.83 & 153.51 & 25093.47 & 0.00 \\
\hline & & 0.1 & 1 & 1 & 0.05 & 0.94 & 1.13 & 20.86 & 153.70 & 25034.76 & -0.23 \\
\hline & & 0.2 & 1 & 1 & 0.05 & 0.94 & 1.14 & 20.96 & 154.47 & 24919.08 & -0.69 \\
\hline & & 0.5 & 1 & 1 & 0.05 & 0.94 & 1.18 & 21.75 & 160.24 & 24586.31 & -2.02 \\
\hline \multirow{5}{*}{$\vec{\varphi}$} & \multirow{5}{*}{$y$} & 0 & 1 & 1 & 0.05 & 0.94 & 1.12 & 20.58 & 153.45 & 25915.05 & 0.23 \\
\hline & & 0.05 & 1 & 1 & 0.05 & 0.94 & 1.12 & 20.59 & 153.51 & 25855.77 & 0.00 \\
\hline & & 0.1 & 1 & 1 & 0.05 & 0.94 & 1.12 & 20.61 & 153.70 & 25797.06 & -0.23 \\
\hline & & 0.2 & 1 & 1 & 0.05 & 0.94 & 1.12 & 20.72 & 154.48 & 25681.37 & -0.67 \\
\hline & & 0.5 & 1 & 1 & 0.05 & 0.94 & 1.17 & 21.49 & 160.24 & 25348.58 & -1.96 \\
\hline \multirow{5}{*}{$\stackrel{\sim}{n}$} & \multirow{5}{*}{$y$} & 0 & 1 & 1 & 0.05 & 0.94 & 0.00 & 21.11 & 153.76 & 24495.27 & 0.24 \\
\hline & & 0.05 & 1 & 1 & 0.05 & 0.94 & 0.00 & 21.12 & 153.83 & 24435.56 & 0.00 \\
\hline & & 0.1 & 1 & 1 & 0.05 & 0.94 & 0.00 & 21.15 & 154.02 & 24376.43 & -0.24 \\
\hline & & 0.2 & 1 & 1 & 0.05 & 0.94 & 0.00 & 21.26 & 154.80 & 24259.90 & -0.72 \\
\hline & & 0.5 & 1 & 1 & 0.05 & 0.94 & 0.00 & 22.05 & 160.60 & 23924.74 & -2.09 \\
\hline
\end{tabular}

As lost sale cost for remanufactured items increases, $Q_{p}^{*}, Q_{s}^{*}$ and $E T P^{*}$ decreases, $Q_{r}^{*}$ increases and the optimal policy shifts to remanufacturing a part of used units collected from the both primary and secondary markets. As lost sale cost for remanufactured items increases, collection rate from primary market increases and from secondary market decreases, that is why depending on the demand for remanufactured and manufactured items, this increased lost sale cost can be beneficial or detrimental for environment.

When the selling price of remanufactured items increases, both $Q_{r}^{*}$ and $E T P^{*}$ increase. When manufacturers can sell remanufactured products at high price, so the minimum allowed quality level is reduced and manufacturers prefer to remanufacture more items to increase their profits. Also as selling price of remanufactured items increases, return rate of used items increases too which is beneficial for environment.

As selling price of newly produced items decreases, $Q_{r}^{*}$ and $E T P^{*}$ will decrease too. When manufacturers can sell newly produced items at high price, at this point, the minimum allowed quality level is increased and manufacturers prefer to produce more new items instead of remanufacturing. Also as selling price of newly produced items increases, return rate of used items decreases which is detrimental for environment.

When $\varphi_{1}$ increases, the expected total profit decreases, but it has a little effect on it. Moreover $Q_{p}^{*}$ and $Q_{s}^{*}$ decrease, $Q_{r}^{*}$ increase and the optimal policy shifts from remanufacturing used items which are collected from the secondary market to remanufacturing a part of used items collected from both markets. When $\varphi_{2}$ increases, the expected total profit increases and $Q_{r}^{*}$ decreases although it has little effect on them, and it has almost no effect on $Q_{p}^{*}$ and optimal strategy. 
Moreover the sensitivity of the model for increasing values of $x$ and $y$ are investigated. The results show that when $x$ and $y$ are increased the expected total profit decreases, although it has a little effect on it. It should be noted that increasing in $y$ has more effect than $x$ on decreasing the expected total profit. Also changes of $x$ and $y$ has almost no effect on the optimal strategy. It is obvious that variations of $x$ and $y$ do not affect environmental issue because return rates and optimal policy of remanufacturing do not change in this situation.
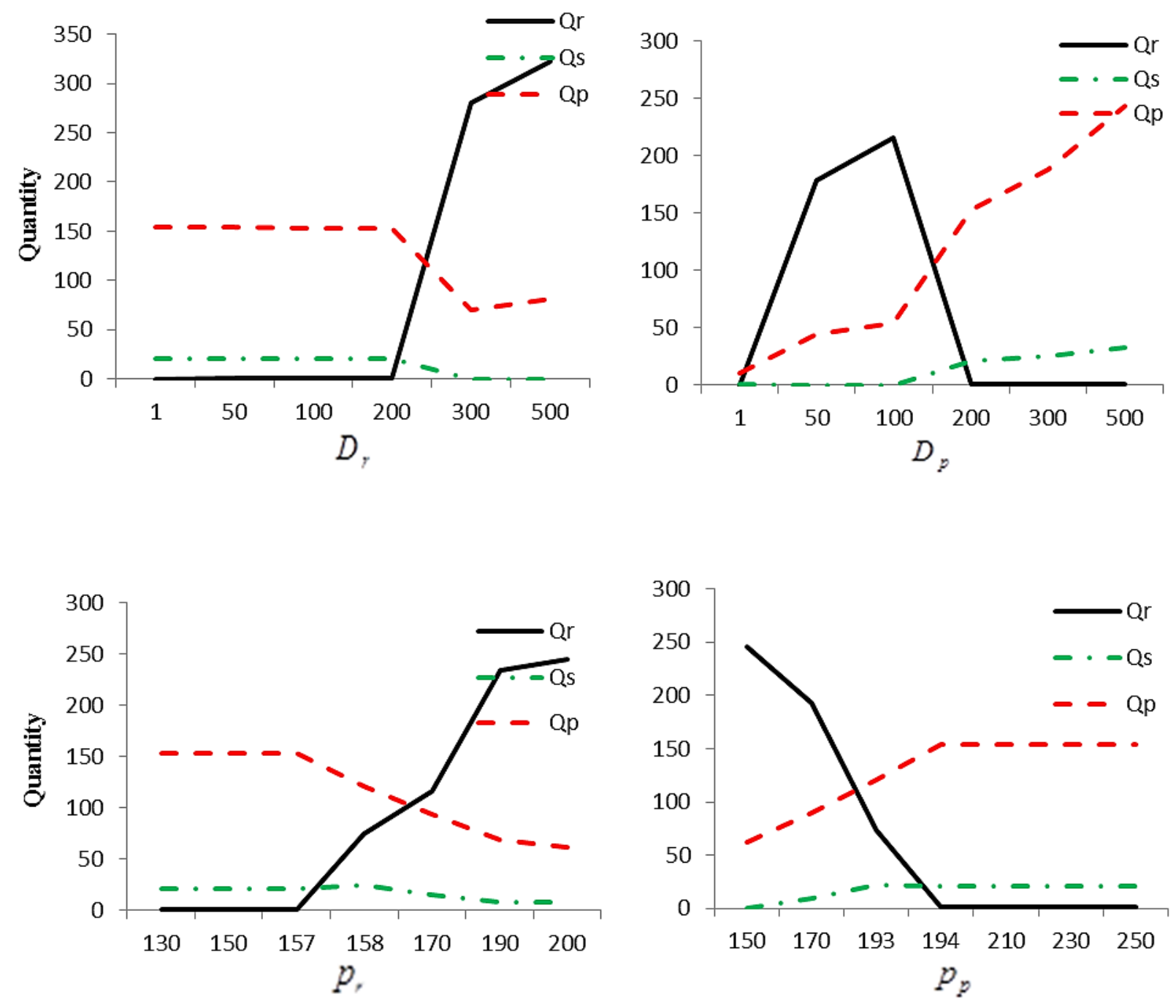

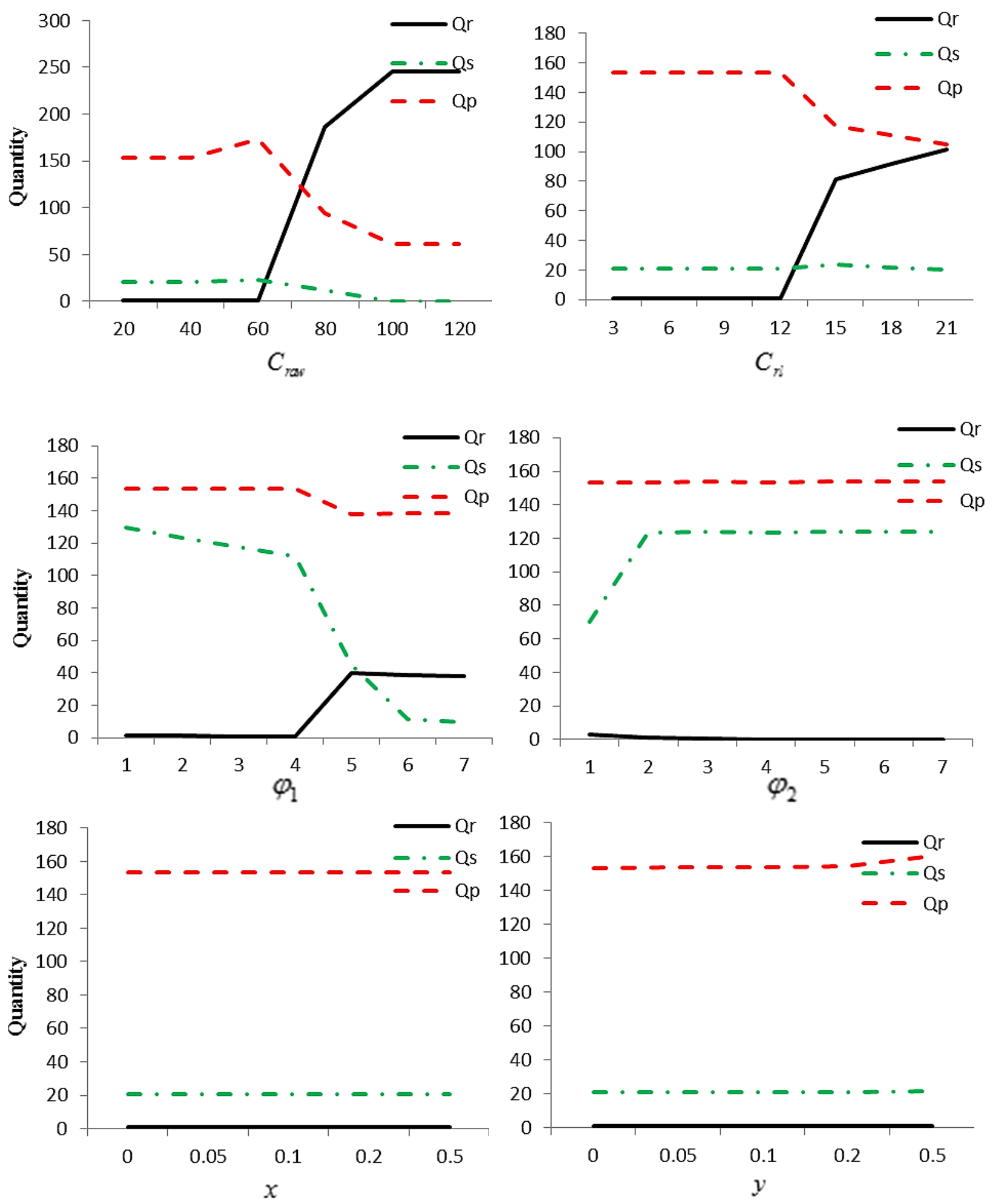

Fig. 6. The changes of $Q_{r}^{*}, Q_{s}^{*}$ and $Q_{p}^{*}$ with respect to the parameters changes 

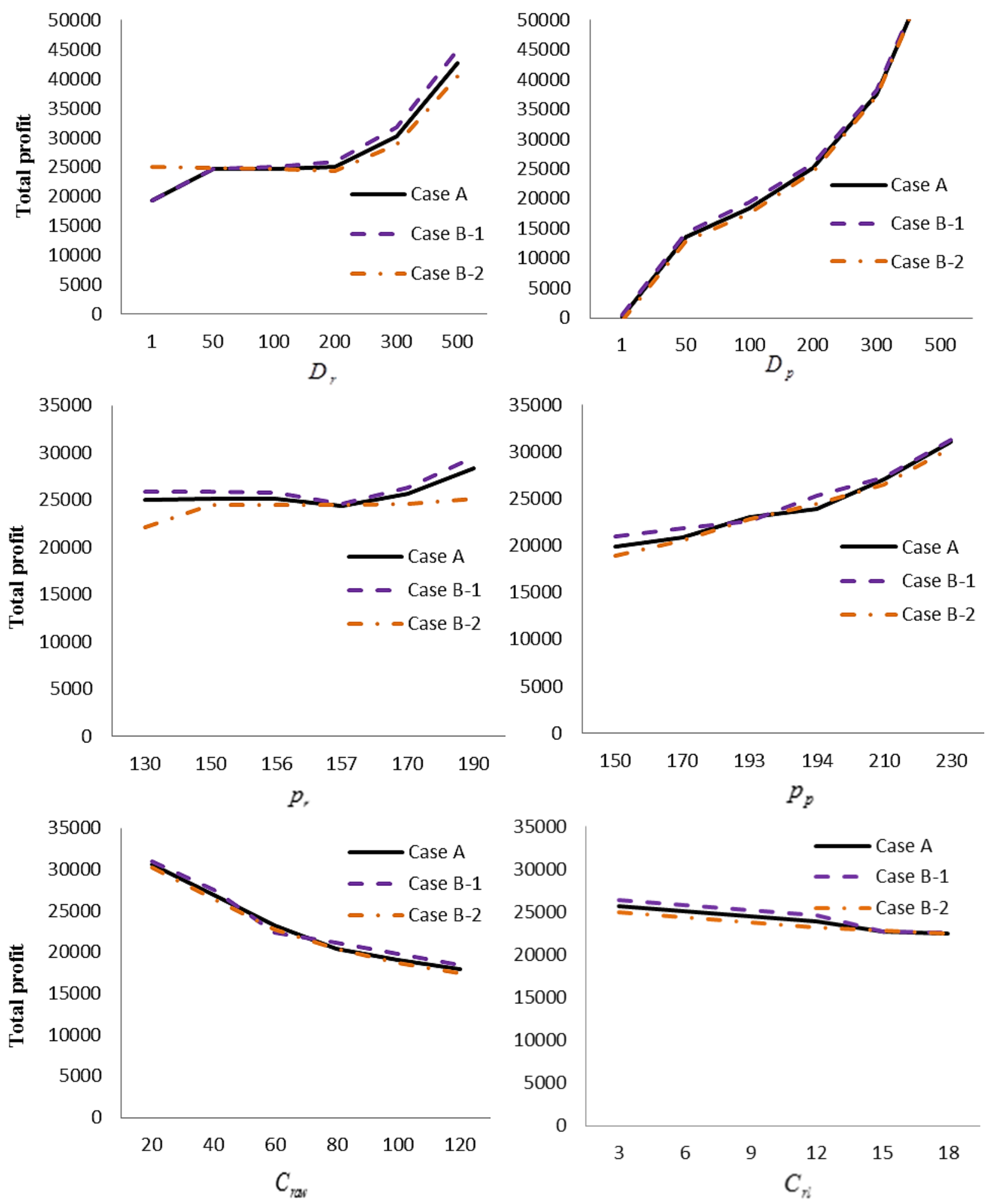

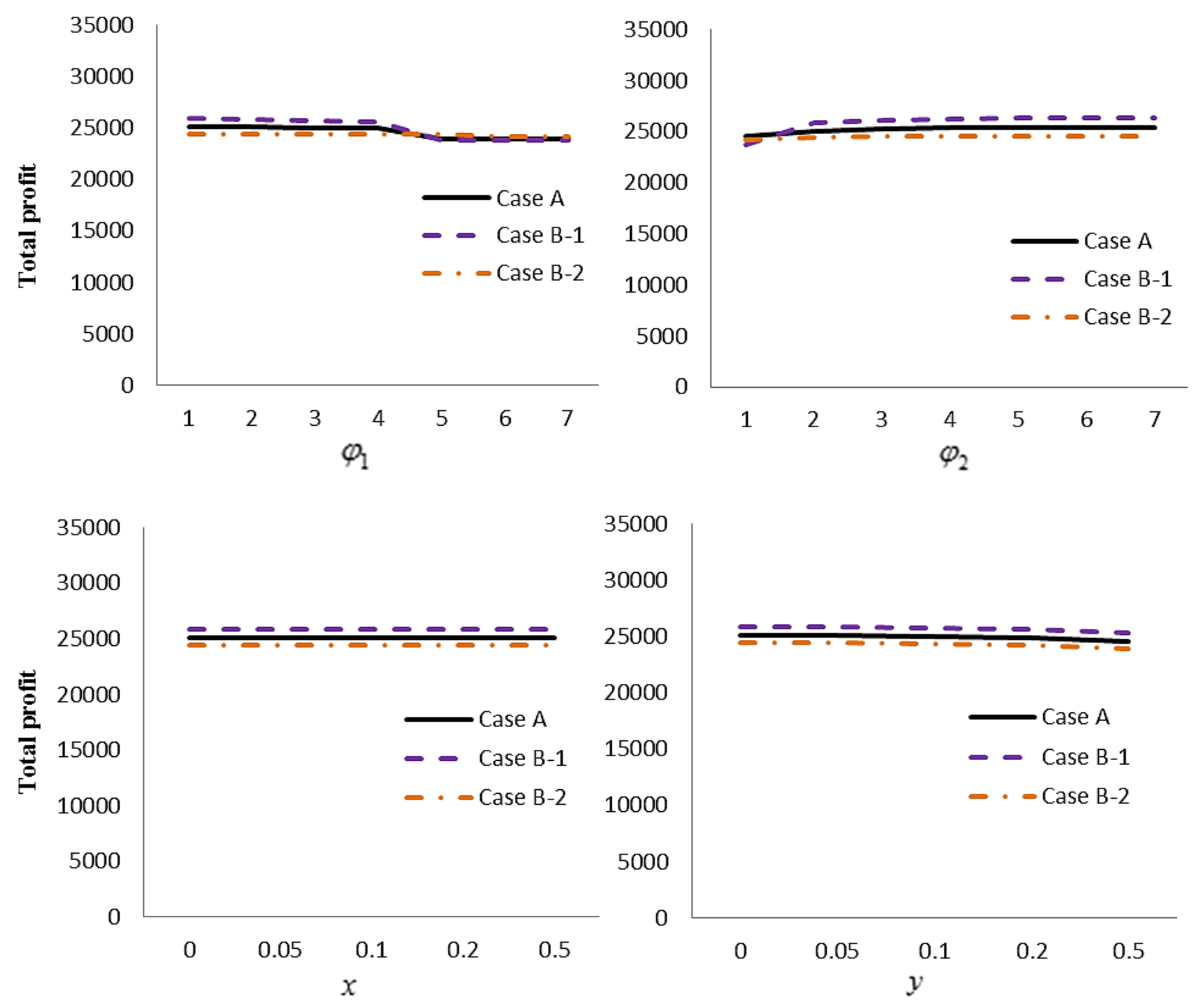

Fig. 7. The changes of expected total profit variables with respect to the parameters changes

\section{Summery and conclusion}

In this paper, we have studied an imperfect production-reproduction system in which the quality of returned items is considered as a random variable and the collection rate of used items is dependent on the acceptance quality level of the returned items. Based on this assumption the buyback costs, remanufacturing costs and salvage values are not same for all returned items. In order to take the randomness of the quality level of returned items into consideration, we have proposed three different probabilistic distribution functions (uniform and simplified triangular P.D.F.s) for quality level of returned items. We also considered a situation where remanufactured items are perceived different from newly produced by customers. This assumption results in lost sales in both manufacturing and remanufacturing periods. In this paper, we proposed a model by which companies can contribute to the environmental issue by remanufacturing and salvaging products instead of disposing them, so this paper can contribute to environmental problems. Also, since the increasing return rate is favorable to the 
CLSC and the environment, companies can contribute to the environmental issue by analyzing the quantity of remanufacturing, salvaging and return rate.

The firm wants to obtain the optimal acceptance quality level of the returns, optimal production, remanufacturing, and salvage quantity, and the optimal number of production and remanufacturing lots. Total profits can be obtained by subtracting the total costs from the total revenue, which is the summation of the total sales of the newly produced and remanufactured items and salvage value of returned items which are not suitable for remanufacturing. The total costs consist of the total purchasing costs of raw materials and returned items, the total set-up costs, the total production and remanufacturing costs, the total holding cost, inspection costs, and reworking costs.

Three mathematical models were developed in the case of multiple remanufacturing and production cycles by considering finite production and remanufacturing rates. In the first, second and third models we assume that the quality of returned items follows the uniform P.D.F., the triangular P.D.F. with ascending behavior and the triangular P.D.F., with descending behavior respectively. After formulating these models, we present a solution procedure to solve these models. The proposed algorithm is programmed in MATLAB and then numerical examples have been presented to show this solution procedure numerically. Finally, sensitivity analysis on the results of numerical examples with respect to some input parameters is conducted from which some results are derived.

The results in numerical examples indicate that the maximum expected profit is obtained for the case of ascending triangular distribution (related to case B.1). It means that, when the probability of returning high quality used items increases, the total expected profit will increase too. So, manufacturers can gain more benefit by the outcomes of the results. According to the results, the optimal recovery policy is almost either to collect all available returns from the primary and secondary markets or to not collect from a primary market but only from the secondary market.

Another result that was obtained in our study is that, as the cost of remanufacturing of one returned item decreases, it is more profitable to satisfy demand for the secondary market rather than the first market. It would be better for the system to collect and remanufacture as many used products as possible. Also in the matter of the environmental impact, companies can examine their firm by analyzing the quantity of remanufacturing, salvaging and return rate. In fact, the increasing return rate is favorable to both the CLSC and the environment because by increasing return rate, larger quantity of used products should be remanufactured or salvaged which can reduce the negative effect of disposal on environment. Therefore, according to sensitivity analysis, increasing the demand for remanufactured, decreasing the demand for newly produced items, increasing the selling price of remanufactured items, and decreasing the selling price of newly produced items are beneficial for environmental issue.

This study can be developed in several ways. For example an interesting extension to this work could be considering that item cannot be remanufactured indefinitely [25]. Another way would be to recast the problem into a multi-buyer or a multi-echelon supply chain. The models of this paper could also be developed by assuming learning in production and remanufacturing [21]. Another interesting development is to account for stochastic demand for each market, random defective rate, or uncertain lead-time, in which holding safety stocks should be considered. Moreover, further investigation can examine the case in which used products are dismantled to recover useful items[23]. This work could also be developed by considering greenhouse gases which are emitted from production and reproduction processes and energy which are used by all processes. 


\section{Acknowledgments}

The second author would like to thank the financial support of University of Tehran for this research under grant number 30015-1-02. This work is supported by Iran National Science Foundation (INSF).

\section{References}

[1] L. E. Cárdenas-Barrón, K.-J. Chung, and G. Treviño-Garza, "Celebrating a century of the economic order quantity model in honor of Ford Whitman Harris," International Journal of Production Economics, vol. 155, pp. 1-7, 2014.

[2] K. Govindan and H. Soleimani, "A review of reverse logistics and closed-loop supply chains: a Journal of Cleaner Production focus," Journal of Cleaner Production, 2016.

[3] M. Fleischmann, J. M. Bloemhof-Ruwaard, R. Dekker, E. Van der Laan, J. A. Van Nunen, and L. N. Van Wassenhove, "Quantitative models for reverse logistics: a review," European journal of operational research, vol. 103, pp. 1-17, 1997.

[4] J. R. Stock and C. J. Broadus, "DOCTORAL RESEARCH IN SUPPLY CHAIN MANAGEMENT AND/OR LOGISTICS-RELATED AREAS: 1999-2004," Journal of Business Logistics, vol. 27, pp. 139-496, 2006.

[5] D. A. Schrady, "A deterministic inventory model for reparable items," Naval Research Logistics Quarterly, vol. 14, pp. 391-398, 1967.

[6] S. NAHMIASJ and H. Rivera, "A deterministic model for a repairable item inventory system with a finite repair rate†," International Journal of Production Research, vol. 17, pp. 215-221, 1979.

[7] M. C. Mabini, L. M. Pintelon, and L. F. Gelders, "EOQ type formulations for controlling repairable inventories," International Journal of Production Economics, vol. 28, pp. 21-33, 1992.

[8] S.-G. Koh, H. Hwang, K.-I. Sohn, and C.-S. Ko, "An optimal ordering and recovery policy for reusable items," Computers \& Industrial Engineering, vol. 43, pp. 59-73, 2002.

[9] K. Richter, "The extended EOQ repair and waste disposal model," International Journal of Production Economics, vol. 45, pp. 443-447, 1996.

[10] K. Richter, "Pure and mixed strategies for the EOQ repair and waste disposal problem," Operations-Research-Spektrum, vol. 19, pp. 123-129, 1997.

[11] K. Richter and I. Dobos, "Analysis of the EOQ repair and waste disposal problem with integer setup numbers," International Journal of Production Economics, vol. 59, pp. 463-467, 3// 1999.

[12] I. Dobos and K. Richter, "An extended production/recycling model with stationary demand and return rates," International Journal of Production Economics, vol. 90, pp. 311-323, 2004.

[13] I. Dobos and K. Richter, "A production/recycling model with quality consideration," International Journal of Production Economics, vol. 104, pp. 571-579, 2006.

[14] R. H. Teunter, "Economic ordering quantities for recoverable item inventory systems," Naval Research Logistics (NRL), vol. 48, pp. 484-495, 2001.

[15] R. Teunter, "Lot-sizing for inventory systems with product recovery," Computers \& Industrial Engineering, vol. 46, pp. 431-441, 2004.

[16] R. Teunter, "Economic ordering quantities for repairable/manufacturable item inventory systems," Preprint, 1998.

[17] I. Dobos and K. Richter, "A production/recycling model with stationary demand and return rates," 2003.

[18] A. El Saadany and M. Y. Jaber, "The EOQ repair and waste disposal model with switching costs," Computers \& Industrial Engineering, vol. 55, pp. 219-233, 2008.

[19] M. Y. Jaber and M. A. Rosen, "The economic order quantity repair and waste disposal model with entropy cost," European Journal of Operational Research, vol. 188, pp. 109-120, 2008.

[20] I. Konstantaras and S. Papachristos, "Lot-sizing for a single-product recovery system with backordering," International Journal of Production Research, vol. 44, pp. 2031-2045, 2006. 
[21] M. Y. Jaber and A. El Saadany, "An economic production and remanufacturing model with learning effects," International Journal of Production Economics, vol. 131, pp. 115-127, 2011.

[22] D.-M. Tsai, "Optimal ordering and production policy for a recoverable item inventory system with learning effect," International Journal of Systems Science, vol. 43, pp. 349-367, 2012.

[23] A. El Saadany and M. Y. Jaber, "A production/remanufacture model with returns' subassemblies managed differently," International Journal of Production Economics, vol. 133, pp. 119-126, 2011.

[24] P. Hasanov, M. Y. Jaber, S. Zanoni, and L. E. Zavanella, "Closed-loop supply chain system with energy, transportation and waste disposal costs," International Journal of Sustainable Engineering, vol. 6, pp. 352-358, 2013/12/01 2013.

[25] A. El Saadany, M. Y. Jaber, and M. Bonney, "How many times to remanufacture?," International Journal of Production Economics, vol. 143, pp. 598-604, 2013.

[26] R. S. Tibben-Lembke and D. S. Rogers, "Differences between forward and reverse logistics in a retail environment," Supply Chain Management: An International Journal, vol. 7, pp. 271-282, 2002.

[27] J. D. Blackburn, V. D. R. Guide, G. C. Souza, and L. N. Van Wassenhove, "Reverse supply chains for commercial returns," California management review, vol. 46, pp. 6-22, 2004.

[28] M. Y. Jaber and A. El Saadany, "The production, remanufacture and waste disposal model with lost sales," International Journal of Production Economics, vol. 120, pp. 115-124, 2009.

[29] I. Konstantaras, K. Skouri, and M. Jaber, "Lot sizing for a recoverable product with inspection and sorting," Computers \& Industrial Engineering, vol. 58, pp. 452-462, 2010.

[30] P. Hasanov, M. Y. Jaber, and S. Zolfaghari, "Production, remanufacturing and waste disposal models for the cases of pure and partial backordering," Applied Mathematical Modelling, vol. 36, pp. 5249-5261, 2012.

[31] S. Singh and N. Saxena, "A closed loop supply chain system with flexible manufacturing and reverse logistics operation under shortages for deteriorating items," Procedia Technology, vol. 10, pp. 330-339, 2013.

[32] A. El Saadany and M. Y. Jaber, "A production/remanufacturing inventory model with price and quality dependant return rate," Computers \& Industrial Engineering, vol. 58, pp. 352-362, 2010.

[33] J. Vörös, "Product balancing under conditions of quality inflation, cost pressures and growth strategies," European Journal of Operational Research, vol. 141, pp. 153-166, 2002.

[34] H. Hwang, Y. D. Ko, S. H. Yune, and C. S. Ko, "A closed-loop recycling system with a minimum allowed quality level on returned products," International Journal of Services and Operations Management, vol. 5, pp. 758-773, 2009.

[35] B. Giri and S. Sharma, "Optimal production policy for a closed-loop hybrid system with uncertain demand and return under supply disruption," Journal of Cleaner Production, vol. 112, pp. 20152028, 2016.

[36] P. Dutta, D. Das, F. Schultmann, and M. Fröhling, "Design and planning of a closed-loop supply chain with three way recovery and buy-back offer," Journal of Cleaner Production, vol. 135, pp. 604-619, 2016.

[37] J. Guo and C. Liang, "Models for recycling and production policy for a closed-loop system," Journal of Quality Vol, vol. 18, p. 303, 2011.

[38] A. Korugan, K. Din, \#231, E. Dinge, \#231, Tolga, et al., "On the quality variation impact of returns in remanufacturing," Comput. Ind. Eng., vol. 64, pp. 929-936, 2013.

[39] J. Guo and G. Ya, "Optimal strategies for manufacturing/remanufacturing system with the consideration of recycled products," Computers \& Industrial Engineering, 2014.

[40] M. R. Galbreth and J. D. Blackburn, "Optimal acquisition and sorting policies for remanufacturing," Production and Operations Management, vol. 15, pp. 384-392, 2006.

[41] L. Xin-jun and D. Qing-li, "Monopoly production optimization problem for remanufacturing cost with stochastic distribution [J]," Control and Decision, vol. 1, p. 008, 2008. 
[42] A. Jamal, B. R. Sarker, and S. Mondal, "Optimal manufacturing batch size with rework process at a single-stage production system," Computers \& Industrial Engineering, vol. 47, pp. 77-89, 2004.

[43] L. E. Cárdenas-Barrón, B. Sarkar, and G. Treviño-Garza, "Easy and improved algorithms to joint determination of the replenishment lot size and number of shipments for an EPQ model with rework," Mathematical and Computational Applications, vol. 18, pp. 132-138, 2013.

[44] L. E. Cárdenas-Barrón, B. Sarkar, and G. Treviño-Garza, "An improved solution to the replenishment policy for the EMQ model with rework and multiple shipments," Applied Mathematical Modelling, vol. 37, pp. 5549-5554, 2013.

[45] B. Sarkar, S. S. Sana, and K. Chaudhuri, "An imperfect production process for time varying demand with inflation and time value of money-An EMQ model," Expert Systems with Applications, vol. 38, pp. 13543-13548, 2011.

[46] H.-M. Wee, W.-T. Wang, and L. E. Cárdenas-Barrón, "An alternative analysis and solution procedure for the EPQ model with rework process at a single-stage manufacturing system with planned backorders," Computers \& Industrial Engineering, vol. 64, pp. 748-755, 2013.

[47] B. Sarkar and I. Moon, "Improved quality, setup cost reduction, and variable backorder costs in an imperfect production process," International Journal of Production Economics, vol. 155, pp. 204-213, 2014.

[48] B. Sarkar, L. E. Cárdenas-Barrón, M. Sarkar, and M. L. Singgih, "An economic production quantity model with random defective rate, rework process and backorders for a single stage production system," Journal of Manufacturing Systems, vol. 33, pp. 423-435, 2014.

[49] G. Treviño-Garza, K. K. Castillo-Villar, and L. E. Cárdenas-Barrón, "Joint determination of the lot size and number of shipments for a family of integrated vendor-buyer systems considering defective products," International Journal of Systems Science, vol. 46, pp. 1705-1716, 2015.

[50] K. Inderfurth*, G. Lindner, and N. Rachaniotis, "Lot sizing in a production system with rework and product deterioration," International Journal of Production Research, vol. 43, pp. 1355-1374, 2005.

[51] B. Sarkar, S. S. Sana, and K. Chaudhuri, "Optimal reliability, production lotsize and safety stock: an economic manufacturing quantity model," International Journal of Management Science and Engineering Management, vol. 5, pp. 192-202, 2010.

[52] B. Sarkar and S. Saren, "Product inspection policy for an imperfect production system with inspection errors and warranty cost," European Journal of Operational Research, vol. 248, pp. 263-271, 2016.

[53] B. Giri and S. Sharma, "Optimizing a closed-loop supply chain with manufacturing defects and quality dependent return rate," Journal of Manufacturing Systems, vol. 35, pp. 92-111, 2015.

[54] S.-S. Chung and C.-S. Poon, "A comparison of waste-reduction practices and new environmental paradigm of rural and urban Chinese citizens," Journal of Environmental Management, vol. 62, pp. 3-19, 2001.

[55] A.A., Taleizadeh, A.A., Najafi A.A., S.T.A., Niaki, (2010a). Multi Product EPQ Model with Scraped Items and limited Production Capacity, International Journal of Science and Technology (Scientia Iranica) Transaction E, vol. 17, pp. 58-69.

[56] A.A., Taleizadeh, S.T.A., Niaki, M.B., Aryanezhad, (2010b). Replenish-up-to Multi ChanceConstraint Inventory Control System with Stochastic Period Lengths and Total Discount under Fuzzy Purchasing Price and Holding Costs, International Journal of System Sciences, vol. 41, pp. $1187-1200$.

[57] A.A., Taleizadeh, S.T.A., Niaki, M.B., Aryanezhad, A., Fallah-Tafti (2010c). A Genetic Algorithm to Optimize Multi-Product Multi-Constraint Inventory Control Systems with Stochastic Replenishments and Discount, International Journal of Advanced Manufacturing Technology, vol. 51, pp. 311-323.

[58] A.A., Taleizadeh, F., Barzinpour, F., H.M., Wee (2011). Meta-heuristic Algorithms to Solve the Fuzzy Single Period Problem, Mathematical and Computer Modeling, vol. 54, pp. 1273-1285. 
[59] A.A., Taleizadeh, D.W., Pentico, M.B., Aryanezhad, M., Ghoreyshi (2012). An Economic Order Quantity Model with Partial Backordering and a Special Sale Price, European Journal of Operational Research, vol. 221, pp. 571-583.

[60] A.A., Taleizadeh, S.T.A., Niaki, M.B., Aryanezhad, N., Shafii (2013a). A Hybrid Method of Fuzzy Simulation and Genetic Algorithm to Optimize Multi-Product Single-Constraint Inventory Control Systems with Stochastic Replenishments and Fuzzy-Demand, Information Science, vol. 220, pp. 425-441.

[61] A.A., Taleizadeh, Pentico D.W., Jabalameli M.S., Aryanezhad M.B., (2013b). An Economic Order Quantity Model with Multiple Partial Prepayments and Partial Backordering, Mathematical and Computer Modeling, vol. 57, pp. 311-323.

[62] A.A., Taleizadeh, H.M., Wee, S.Gh.R., Jalali-Naini (2013c). Economic production quantity model with repair failure and limited capacity, Applied Mathematical Modeling, vol. 37, pp. 2765-2774.

[63] A.A., Taleizadeh, B., Mohammadi, L.E., Cárdenas-Barron, H., Samimi (2013e). An EOQ model for perishable product with special sale and shortage, International Journal of Production Economics, vol. 145, pp. 318-338.

[64] A.A., Taleizadeh, L.E., Cardenas-Barron, B., Mohammadi, (2014). Multi-Product Single Machine EPQ model with Backordering, Scraped Products, Rework and Interruption in Manufacturing Process. International Journal of Production Economic, vol. 150, pp. 9-27.

[65] A.A., Taleizadeh, M., Nouri-Dariyan, L.E., Cárdenas-Barrón (2015a). Joint optimization of price, replenishment frequency, replenishment cycle and production rate in vendor managed inventory system with deteriorating items. International Journal of Production Economics, vol. 159, pp. 285-295.

[66] A.A., Taleizadeh, D.W., Pentico (2013d). An economic order quantity model with known price increase and partial backordering. European Journal of Operational Research, vol. 28, pp. 516525 .

[67] B., Mohammadi, A.A., Taleizadeh, R., Noorossana, H., Samimi, (2015b). Optimizing integrated manufacturing and products inspection policy for deteriorating manufacturing system with imperfect inspection, Journal of Manufacturing Systems, vol. 37, pp. 299-315.

Appendix A. Computing holding cost of returned items

For computing $H_{u}$, the area is divided into $m A$ and $m B$ triangles, a single $\mathrm{C}$ trapezoid and $(m-1) D$ squares, as shown in Fig. 8. 


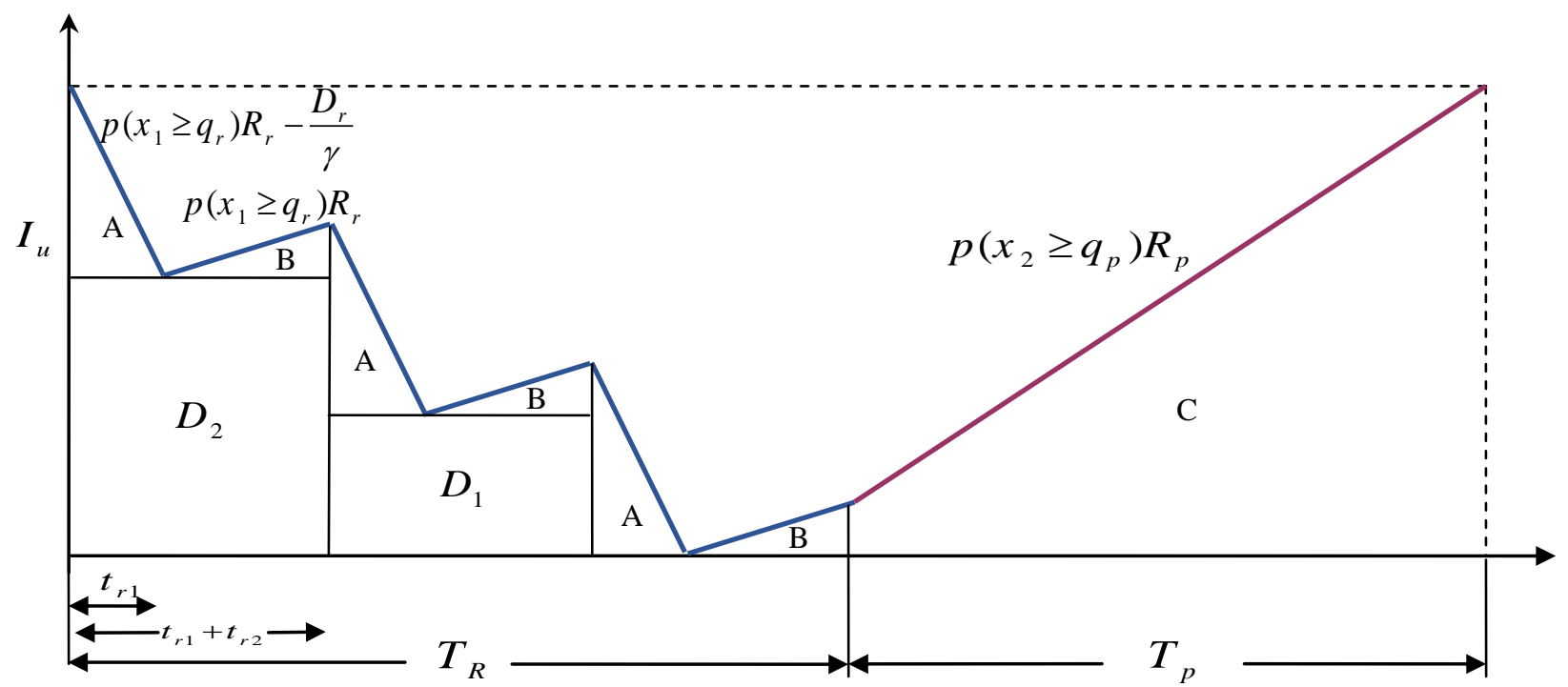

Fig. 8. Inventory level of the returned stocks

Area of triangle A is:

$S_{A}=\frac{t_{r 1}^{2}}{2}\left(\frac{D_{r}}{\gamma}-p\left(x_{1} \geq q_{r}\right) R_{r}\right)=\frac{D_{r} T^{2}}{2}\left(\frac{\gamma \lambda^{2}}{m^{2}}\left(1-\gamma \alpha_{r} p\left(x_{1} \geq q_{r}\right)\right)\right)$

Area of triangle $\mathrm{B}$ is:

$S_{B}=\frac{\left(t_{r 2}+t_{r 3}\right)^{2}}{2}\left(R_{r} p\left(x_{1} \geq q_{r}\right)\right)=\frac{D_{r} T^{2}}{2}\left(\frac{(1-\gamma)^{2} \lambda^{2}}{m^{2}}\left(\alpha_{r} p\left(x_{1} \geq q_{r}\right)\right)\right)$

Area of trapezoid $\mathrm{C}$ is:

$$
\begin{aligned}
S_{c} & =\frac{1}{2}\left(2\left(t_{r 2}+t_{r 3}\right)\left(R_{r} p\left(x_{1} \geq q_{r}\right)\right)+\left(T_{p}\right)\left(R_{p} p\left(x_{2} \geq q_{p}\right)\right)\right) T_{p} \\
& =\frac{D_{r} T^{2}}{2}\left(2 \alpha_{r} p\left(x_{1} \geq q_{r}\right)\left(\frac{(1-\gamma) \lambda(1-\lambda)}{m}\right)\right)+\frac{D_{p} T^{2}}{2}\left(\alpha_{p} p\left(x_{2} \geq q_{p}\right)(1-\lambda)^{2}\right)
\end{aligned}
$$

Area of square $D_{i}$ is:

$S_{D_{i}}=\left[\left(\frac{D_{r}}{\gamma}-R_{r} p\left(x_{1} \geq q_{r}\right)\right) t_{r 1}-R_{r} p\left(x_{1} \geq q_{r}\right)\left(t_{r 2}+t_{r 3}\right)\right] \frac{\lambda T}{m} \cdot i=\frac{D_{r}}{T^{2}}\left(\frac{\lambda^{2}}{m^{2}}\left(1-\alpha_{r} p\left(x_{1} \geq q_{r}\right)\right)\right) i$

So, the average inventory level of returned items can be expressed as below:

$\overline{I_{u}}=m S_{A}+m S_{B}+S_{c}+\sum_{i=1}^{m-1} S_{D_{i}}$ 


$$
\begin{aligned}
\overline{I_{u}}= & \frac{1}{2} D_{r} T^{2}\left[\begin{array}{l}
\frac{\gamma \lambda^{2}}{m}\left(1-\gamma \alpha_{r} p\left(x_{1} \geq q_{r}\right)\right)+\frac{(1-\gamma)^{2} \lambda^{2} \alpha_{r} p\left(x_{1} \geq q_{r}\right)}{m}+\frac{(m-1) \lambda^{2}}{m}\left(1-\alpha_{r} p\left(x_{1} \geq q_{r}\right)\right) \\
+2 \alpha_{r} p\left(x_{1} \geq q_{r}\right) \frac{(1-\gamma) \lambda(1-\lambda)}{m}
\end{array}\right] \\
& +\frac{1}{2} D_{p} T^{2}\left[\alpha_{p} p\left(x_{2} \geq q_{p}\right)(1-\lambda)^{2}\right]
\end{aligned}
$$

Therefore, the inventory holding cost of returned items can be obtained as follows:

$$
\begin{aligned}
H_{u}=h_{u}\left(\overline{I_{u}}\right) & =\frac{1}{2} D_{r} h_{u} T^{2}\left[\begin{array}{l}
\frac{\gamma \lambda^{2}}{m}\left(1-\gamma \alpha_{r} p\left(x_{1} \geq q_{r}\right)\right)+\frac{(1-\gamma)^{2} \lambda^{2} \alpha_{r} p\left(x_{1} \geq q_{r}\right)}{m}+\frac{(m-1) \lambda^{2}}{m}\left(1-\alpha_{r} p\left(x_{1} \geq q_{r}\right)\right) \\
+2 \alpha_{r} p\left(x_{1} \geq q_{r}\right) \frac{(1-\gamma) \lambda(1-\lambda)}{m}
\end{array}\right] \\
& +\frac{1}{2} D_{p} h_{u} T^{2}\left[\alpha_{p} p\left(x_{2} \geq q_{p}\right)(1-\lambda)^{2}\right]
\end{aligned}
$$

Appendix B. Computing holding cost of raw materials

Raw material stock

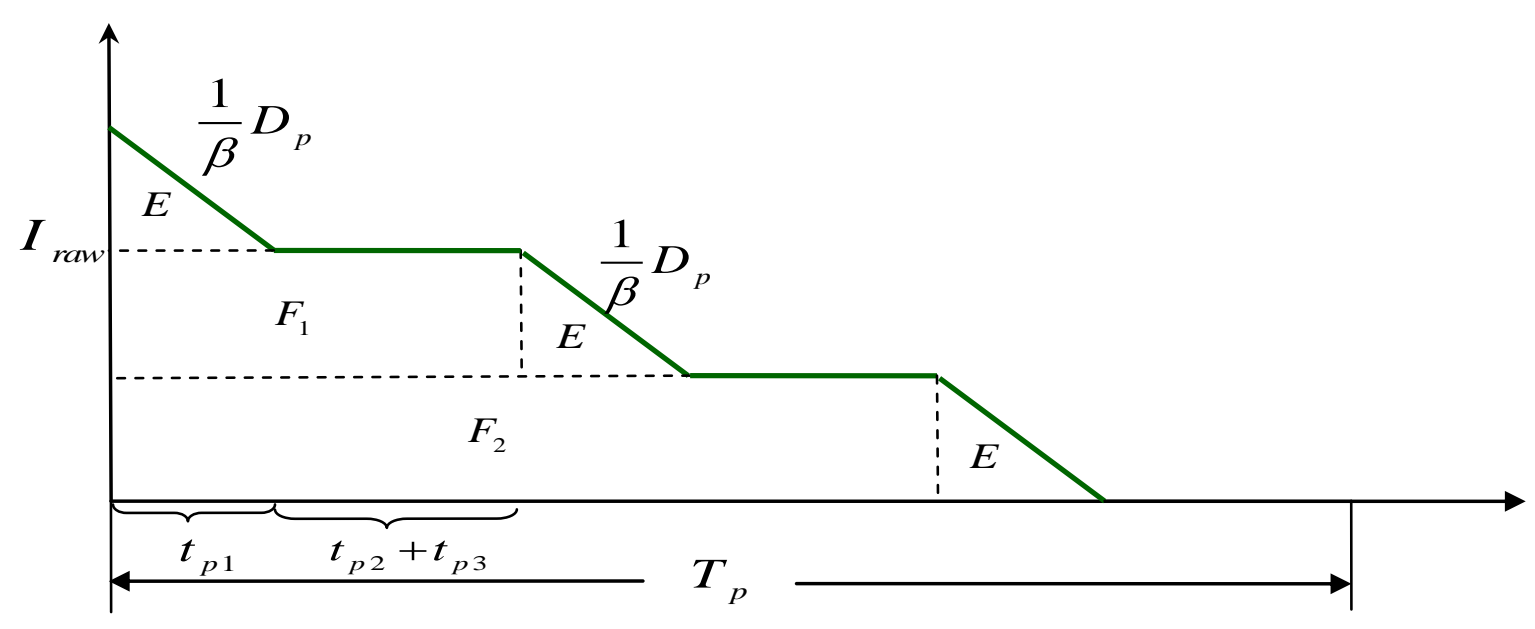

Fig. 9. Inventory level of the raw material

For computing $H_{\text {raw }}$, the area is divided into $n E$ triangles and $(n-1) D$ squares, as shown in Fig. 9. Area of triangle $\mathrm{E}$ is:

$S_{E}=\frac{t_{p 1}^{2}}{2}\left(\frac{1}{\beta} D_{p}\right)=\frac{D_{p} T^{2}}{2}\left(\frac{\beta(1-\lambda)^{2}}{n^{2}}\right)$ 
Area of square $F_{i}$ is:

$S_{F_{i}}=\left(\frac{D_{p}}{\beta} t_{p 1}\right) T_{p} . i=\left(\frac{D_{p}}{\beta} t_{p 1}\right) \cdot \frac{(1-\lambda) T}{n} \cdot i=D_{p} T^{2}\left(\frac{(1-\lambda)^{2}}{n^{2}} . i\right)$

So, the average inventory level of raw material can be expressed as follows:

$\overline{I_{\text {raw }}}=n S_{E}+\sum_{i=1}^{n-1} S_{F_{i}}$
$\overline{I_{\text {raw }}}=\frac{1}{2} D_{p} T^{2}\left[\frac{(1-\lambda)^{2}(\beta+n-1)}{n}\right]$

Therefore, the inventory holding cost of returned items can be obtained as follows:

$$
H_{\text {raw }}=h_{\text {raw }}\left(\overline{I_{\text {raw }}}\right)=\frac{1}{2} h_{\text {raw }} D_{p} T^{2} \frac{(1-\lambda)^{2}}{n}(\beta+n-1)=\frac{1}{2} D_{p} T^{2} \psi_{p}^{\text {raw }}
$$

Appendix C. Proof of Theorem.1

Taking the first and the second derivatives of ETP with respect to $T$, we obtain:

$$
\begin{aligned}
& \frac{\partial E T P}{\partial T}=\frac{A}{T^{2}}+\frac{S}{T^{2}}-\frac{D_{r} \psi_{r}}{2}-\frac{D_{p} \psi_{p}}{2} \\
& \frac{\partial^{2} E T P}{\partial T^{2}}=-\frac{A}{T^{3}}-\frac{S}{T^{3}}
\end{aligned}
$$

Since $\partial^{2} E T P / \partial T^{2}<0 ; \forall T>0$, the total expected profit is a concave function over $T$.

Appendix D. Figures of distribution functions related to Case A, B.1, and B.2

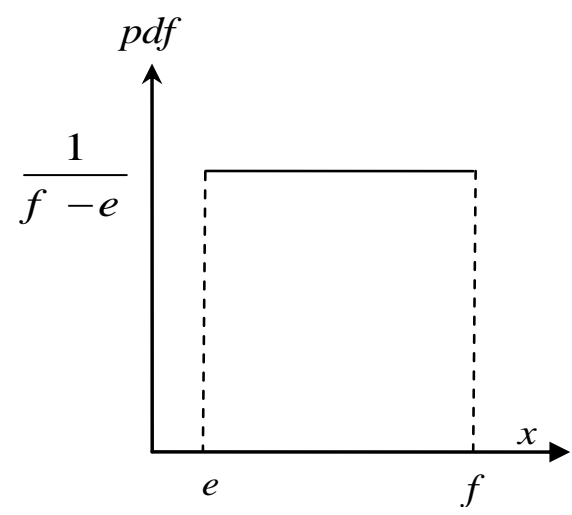

Fig. 10. Uniform distribution function related to case $\mathrm{A}$

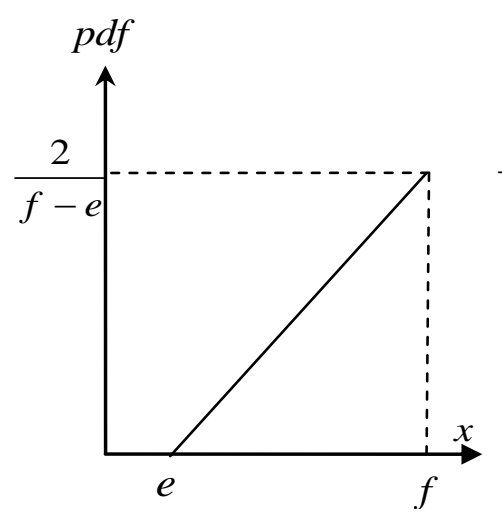

Fig. 10. Triangular distribution function related to case B.1

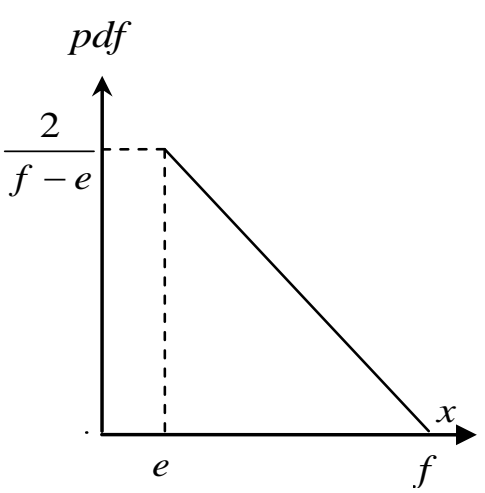

Fig. 12. Triangular distribution function related to case B.2 
Appendix E. Calculations of the average remanufacturing cost ratio, buyback cost ratio, sales revenue ratios, and the probability of remanufacturing or salvaging a returned item for case A

By considering $x_{1}$ and $x_{2}$ as a stochastic variable following uniform P.D.F, $p\left(x_{1} \geq q_{r}\right), p\left(x_{2} \geq q_{p}\right)$, $p\left(x_{1} \leq q_{r}\right), p\left(x_{2} \leq q_{p}\right)$, can be calculated easily as below;

$p\left(x_{1} \geq q_{r}\right)=\frac{f_{1}-q_{r}}{f_{1}-e_{1}}$

$p\left(x_{2} \geq q_{p}\right)=\frac{f_{2}-q_{p}}{f_{2}-e_{2}}$

$p\left(x_{1} \leq q_{r}\right)=\frac{q_{r}-e_{1}}{f_{1}-e_{1}}$

$p\left(x_{2} \leq q_{p}\right)=\frac{q_{p}-e_{2}}{f_{2}-e_{2}}$

The average remanufacturing cost ratio of returned items which are suitable for remanufacturing (related to different quality level $x$ ) can be written as follows:

$$
\begin{aligned}
& E\left(C_{r r}\right)=\int_{q_{r}}^{1} f\left(x_{1}\right) c_{1} e^{\delta_{1}\left(1-x_{1}\right)} d x_{1}=\int_{q_{r}}^{f_{1}} \frac{1}{f_{1}-e_{1}} c_{1} e^{\delta_{1}\left(1-x_{1}\right)} d x_{1}=\frac{c_{1} e^{\delta_{1}}}{\delta_{1}\left(f_{1}-e_{1}\right)}\left[e^{-\delta_{1} q_{r}}-e^{-\delta_{1} f_{1}}\right] \\
& E\left(C_{r p}\right)=\int_{q_{p}}^{1} f\left(x_{2}\right) c_{2} e^{\delta_{2}\left(1-x_{2}\right)} d x_{2}=\int_{q_{p}}^{f_{2}} \frac{1}{f_{2}-e_{2}} a_{2} e^{-\theta_{2}\left(1-x_{2}\right)} d x_{2}=\frac{c_{2} e^{\delta_{2}}}{\delta_{2}\left(f_{2}-e_{2}\right)}\left[e^{-\delta_{2} q_{p}}-e^{-\delta_{2} f_{2}}\right]
\end{aligned}
$$

Moreover for the average buyback cost ratio we have:

$$
\begin{aligned}
& E\left(C_{b r}\right)=\int_{-\infty}^{+\infty} f\left(x_{1}\right) a_{1} e^{-\theta_{1}\left(1-x_{1}\right)} d x_{1}=\int_{e_{1}}^{f_{1}} \frac{1}{f_{1}-e_{1}} a_{1} e^{-\theta_{1}\left(1-x_{1}\right)} d x_{1}=\frac{a_{1} e^{-\theta_{1}}}{\theta_{1}\left(f_{1}-e_{1}\right)}\left[e^{\theta_{1} f_{1}}-e^{\theta_{1} e_{1}}\right] \\
& E\left(C_{b p}\right)=\int_{-\infty}^{+\infty} f\left(x_{2}\right) a_{2} e^{-\theta_{2}\left(1-x_{2}\right)} d x_{2}=\int_{e_{2}}^{f_{2}} \frac{1}{f_{2}-e_{2}} a_{2} e^{-\theta_{2}\left(1-x_{2}\right)} d x_{2}=\frac{a_{2} e^{-\theta_{2}}}{\theta_{2}\left(f_{2}-e_{2}\right)}\left[e^{\theta_{2} f_{2}}-e^{\theta_{2} e_{2}}\right]
\end{aligned}
$$

In the other hand the average sales revenue ratios for returned items which are not suitable for remanufacturing are given by:

$$
\begin{aligned}
& E\left(p_{s r}\right)=\int_{0}^{q_{r}} f\left(x_{1}\right) a_{1} e^{-\theta_{1}\left(1-x_{1}\right)} d x_{1}=\int_{e_{1}}^{q_{r}} \frac{1}{f_{1}-e_{1}} a_{1} e^{-\theta_{1}\left(1-x_{1}\right)} d x_{1}=\frac{a_{1} e^{-\theta_{1}}}{\theta_{1}\left(f_{1}-e_{1}\right)}\left[e^{\theta_{1} q_{r}}-e^{\theta_{1} e_{1}}\right] \\
& E\left(p_{s p}\right)=\int_{0}^{q_{p}} f\left(x_{2}\right) a_{2} e^{-\theta_{2}\left(1-x_{2}\right)} d x_{2}=\int_{e_{2}}^{q_{p}} \frac{1}{f_{2}-e_{2}} a_{2} e^{-\theta_{1}\left(1-x_{1}\right)} d x_{2}=\frac{a_{2} e^{-\theta_{2}}}{\theta_{2}\left(f_{2}-e_{2}\right)}\left[e^{\theta_{2} q_{p}}-e^{\theta_{2} e_{2}}\right]
\end{aligned}
$$


Appendix F. Calculations of the average remanufacturing cost ratio, buyback cost ratio, sales revenue ratios, and the probability of remanufacturing or salvaging a returned item for case B.1

By considering $x_{1}$ and $x_{2}$ as a stochastic variable following triangular PDF related to case B.1, $p\left(x_{1} \geq q_{r}\right), \quad p\left(x_{2} \geq q_{p}\right), p\left(x_{1} \leq q_{r}\right), p\left(x_{2} \leq q_{p}\right)$, can be calculated easily as follows:

$$
\begin{aligned}
& p\left(x_{1} \geq q_{r}\right)=1-\frac{\left(q_{r}-e_{1}\right)^{2}}{\left(f_{1}-e_{1}\right)^{2}} \\
& p\left(x_{2} \geq q_{p}\right)=1-\frac{\left(q_{p}-e_{2}\right)^{2}}{\left(f_{2}-e_{2}\right)^{2}} \\
& p\left(x_{1} \leq q_{r}\right)=\frac{\left(q_{r}-e_{1}\right)^{2}}{\left(f_{1}-e_{1}\right)^{2}} \\
& p\left(x_{2} \leq q_{p}\right)=\frac{\left(q_{p}-e_{2}\right)^{2}}{\left(f_{2}-e_{2}\right)^{2}}
\end{aligned}
$$

The average buyback cost ratio can be written as follows:

$$
\begin{aligned}
E\left(C_{b r}\right) & =\int_{e_{1}}^{f_{1}} \frac{2\left(x_{1}-e_{1}\right)}{\left(f_{1}-e_{1}\right)^{2}} a_{1} e^{-\theta_{1}\left(1-x_{1}\right)} d x_{1} \\
& =\frac{2 a_{1} e_{1}\left(e^{\theta_{1}\left(e_{1}-1\right)}-e^{\theta_{1}\left(f_{1}-1\right)}\right)}{\theta_{1}\left(f_{1}-e_{1}\right)^{2}}-\frac{2 a_{1}\left(\left(e_{1} \theta_{1}-1\right) e^{\theta_{1}\left(e_{1}-1\right)}-\left(f_{1} \theta_{1}-1\right) e^{\theta_{1}\left(f_{1}-1\right)}\right)}{\theta_{1}^{2}\left(f_{1}-e_{1}\right)^{2}} \\
E\left(C_{b p}\right) & =\int_{e_{2}}^{f_{2}} \frac{2\left(x_{2}-e_{2}\right)}{\left(f_{2}-e_{2}\right)^{2}} a_{2} e^{-\theta_{2}\left(1-x_{2}\right)} d x_{2} \\
& =\frac{2 a_{2} e_{2}\left(e^{\theta_{2}\left(e_{2}-1\right)}-e^{\theta_{2}\left(f_{2}-1\right)}\right)}{\theta_{2}\left(f_{2}-e_{2}\right)^{2}}-\frac{2 a_{2}\left(\left(e_{2} \theta_{2}-1\right) e^{\theta_{2}\left(e_{2}-1\right)}-\left(f_{2} \theta_{2}-1\right) e^{\theta_{2}\left(f_{2}-1\right)}\right)}{\theta_{2}^{2}\left(f_{2}-e_{2}\right)^{2}}
\end{aligned}
$$

The average remanufacturing cost ratios of returned items which are suitable for remanufacturing can be obtained as below:

$$
\begin{aligned}
E\left(C_{r r}\right) & =\int_{q_{r}}^{f_{1}} \frac{2\left(x_{1}-e_{1}\right)}{\left(f_{1}-e_{1}\right)^{2}} c_{1} e^{\delta_{1}\left(1-x_{1}\right)} d x_{1} \\
= & \frac{\left[2 c_{1}\left(\left(\delta_{1} q_{r}+1\right) e^{\delta_{1}\left(1-q_{r}\right)}-\left(\delta_{1} f_{1}+1\right) e^{\delta_{1}\left(1-f_{1}\right)}\right)\right]}{\delta_{1}^{2}\left(e_{1}-f_{1}\right)^{2}}+\frac{\left[2 c_{1} e_{1}\left(\frac{1}{e^{\delta_{1}\left(f_{1}-1\right)}}-\frac{1}{e^{\delta_{1}\left(q_{r}-1\right)}}\right)\right]}{\delta_{1}\left(e_{1}-f_{1}\right)^{2}} \\
E\left(C_{r p}\right) & =\int_{q_{p}}^{f_{2}} \frac{2\left(x_{2}-e_{2}\right)}{\left(f_{2}-e_{2}\right)^{2}} c_{2} e^{\delta_{2}\left(1-x_{2}\right)} d x_{2} \\
& =\frac{\left[2 c_{2}\left(\left(\delta_{2} q_{p}+1\right) e^{\delta_{2}\left(1-q_{p}\right)}-\left(\delta_{2} f_{2}+1\right) e^{\delta_{2}\left(1-f_{2}\right)}\right)\right]}{\delta_{2}^{2}\left(e_{2}-f_{2}\right)^{2}}+\frac{\left[2 c_{2} e_{2}\left(\frac{1}{e^{\delta_{2}\left(f_{2}-1\right)}}-\frac{1}{e^{\delta_{2}\left(q_{p}-1\right)}}\right)\right]}{\delta_{2}\left(e_{2}-f_{2}\right)^{2}}
\end{aligned}
$$


Moreover the average sales revenue ratios for returned items which are not suitable for remanufacturing are:

$$
\begin{aligned}
E\left(p_{s r}\right) & =\int_{e_{1}}^{q_{r}} \frac{2\left(x_{1}-e_{1}\right)}{\left(f_{1}-e_{1}\right)^{2}} a_{1} e^{-\theta_{1}\left(1-x_{1}\right)} d x_{1} \\
& =\frac{2 a_{1} e_{1}\left(e^{\theta_{1}\left(q_{r}-1\right)}-e^{\theta_{1}\left(e_{1}-1\right)}\right)}{\theta_{1}\left(f_{1}-e_{1}\right)^{2}}-\frac{2 a_{1}\left(\left(e_{1} \theta_{1}-1\right) e^{\theta_{1}\left(e_{1}-1\right)}-\left(q_{r} \theta_{1}-1\right) e^{\theta_{1}\left(q_{r}-1\right)}\right)}{\theta_{1}^{2}\left(f_{1}-e_{1}\right)^{2}} \\
E\left(p_{s p}\right) & =\int_{e_{2}}^{q_{p}} \frac{2\left(x_{2}-e_{2}\right)}{\left(f_{2}-e_{2}\right)^{2}} a_{2} e^{-\theta_{2}\left(1-x_{2}\right)} d x_{2} \\
& =\frac{2 a_{2} e_{2}\left(e^{\theta_{2}\left(q_{p}-1\right)}-e^{\theta_{2}\left(e_{2}-1\right)}\right)}{\theta_{2}\left(f_{2}-e_{2}\right)^{2}}-\frac{2 a_{2}\left(\left(e_{2} \theta_{2}-1\right) e^{\theta_{2}\left(e_{2}-1\right)}-\left(q_{p} \theta_{2}-1\right) e^{\theta_{2}\left(q_{p}-1\right)}\right)}{\theta_{2}^{2}\left(f_{2}-e_{2}\right)^{2}}
\end{aligned}
$$

Appendix G. Calculations of the average remanufacturing cost ratio, buyback cost ratio, sales revenue ratios, and the probability of remanufacturing or salvaging a returned item for case B.1

By considering $x_{1}$ and $x_{2}$ as a stochastic variable following triangular PDF related to case B.2, $p\left(x_{1} \geq q_{r}\right), p\left(x_{2} \geq q_{p}\right), p\left(x_{1} \leq q_{r}\right), p\left(x_{2} \leq q_{p}\right)$, can be calculated easily as following:

$$
\begin{aligned}
& p\left(x_{1} \geq q_{r}\right)=\frac{\left(f_{1}-q_{r}\right)^{2}}{\left(f_{1}-e_{1}\right)^{2}} \\
& p\left(x_{2} \geq q_{p}\right)=\frac{\left(f_{2}-q_{p}\right)^{2}}{\left(f_{2}-e_{2}\right)^{2}} \\
& p\left(x_{1} \leq q_{r}\right)=1-\frac{\left(f_{1}-q_{r}\right)^{2}}{\left(f_{1}-e_{1}\right)^{2}} \\
& p\left(x_{2} \leq q_{p}\right)=1-\frac{\left(f_{2}-q_{p}\right)^{2}}{\left(f_{2}-e_{2}\right)^{2}}
\end{aligned}
$$

The average buyback cost ratio can be expressed as follows:

$$
\begin{aligned}
E\left(C_{b r}\right) & =\int_{e_{1}}^{f_{1}} \frac{2\left(f_{1}-x_{1}\right)}{\left(f_{1}-e_{1}\right)^{2}} a_{1} e^{-\theta_{1}\left(1-x_{1}\right)} d x_{1} \\
& =\frac{2 a_{1} f_{1}\left(e^{\theta_{1}\left(f_{1}-1\right)}-e^{\theta_{1}\left(e_{1}-1\right)}\right)}{\theta_{1}\left(f_{1}-e_{1}\right)^{2}}+\frac{2 a_{1}\left(\left(e_{1} \theta_{1}-1\right) e^{\theta_{1}\left(e_{1}-1\right)}-\left(f_{1} \theta_{1}-1\right) e^{\theta_{1}\left(f_{1}-1\right)}\right)}{\theta_{1}^{2}\left(f_{1}-e_{1}\right)^{2}} \\
E\left(C_{b p}\right) & =\int_{e_{2}}^{f_{2}} \frac{2\left(f_{2}-x_{2}\right)}{\left(f_{2}-e_{2}\right)^{2}} a_{2} e^{-\theta_{2}\left(1-x_{2}\right)} d x_{2} \\
& =\frac{2 a_{2} f_{2}\left(e^{\theta_{2}\left(f_{2}-1\right)}-e^{\theta_{2}\left(e_{2}-1\right)}\right)}{\theta_{2}\left(f_{2}-e_{2}\right)^{2}}+\frac{2 a_{2}\left(\left(e_{2} \theta_{2}-1\right) e^{\theta_{2}\left(e_{2}-1\right)}-\left(f_{2} \theta_{2}-1\right) e^{\theta_{2}\left(f_{2}-1\right)}\right)}{\theta_{2}^{2}\left(f_{2}-e_{2}\right)^{2}}
\end{aligned}
$$

Moreover the average remanufacturing cost ratios of returned items which are suitable for remanufacturing can be obtained as below: 


$$
\begin{aligned}
E\left(C_{r r}\right) & =\int_{q_{r}}^{f_{1}} \frac{2\left(f_{1}-x_{1}\right)}{\left(f_{1}-e_{1}\right)^{2}} c_{1} e^{\delta_{1}\left(1-x_{1}\right)} d x_{1} \\
= & \frac{\left[2 c_{1}\left(\left(\delta_{1} f_{1}+1\right) e^{\delta_{1}\left(1-f_{1}\right)}-\left(\delta_{1} q_{r}+1\right) e^{\delta_{1}\left(1-q_{r}\right)}\right)\right]}{\delta_{1}^{2}\left(e_{1}-f_{1}\right)^{2}}-\frac{\left[2 c_{1} f_{1}\left(\frac{1}{e^{\delta_{1}\left(f_{1}-1\right)}}-\frac{1}{e^{\delta_{1}\left(q_{r}-1\right)}}\right)\right]}{\delta_{1}\left(e_{1}-f_{1}\right)^{2}} \\
E\left(C_{r p}\right) & =\int_{q_{p}}^{f_{2}} \frac{2\left(f_{2}-x_{2}\right)}{\left(f_{2}-e_{2}\right)^{2}} c_{2} e^{\delta_{2}\left(1-x_{2}\right)} d x_{2} \\
& =\frac{\left[2 c_{2}\left(\left(\delta_{2} f_{2}+1\right) e^{\delta_{2}\left(1-f_{2}\right)}-\left(\delta_{2} q_{p}+1\right) e^{\delta_{2}\left(1-q_{p}\right)}\right)\right]}{\delta_{2}^{2}\left(e_{2}-f_{2}\right)^{2}}-\frac{\left[2 c_{2} f_{2}\left(\frac{1}{e^{\delta_{2}\left(f_{2}-1\right)}}-\frac{1}{e^{\delta_{2}\left(q_{p}-1\right)}}\right)\right]}{\delta_{2}\left(e_{2}-f_{2}\right)^{2}}
\end{aligned}
$$

The average sales revenue ratios of returned items which are not suitable for remanufacturing are:

$$
\begin{aligned}
E\left(p_{s r}\right) & =\int_{0}^{q_{r}} f\left(x_{1}\right) a_{1} e^{-\theta_{1}\left(1-x_{1}\right)} d x_{1}=\int_{e_{1}}^{q_{r}} \frac{2\left(f_{1}-x_{1}\right)}{\left(f_{1}-e_{1}\right)^{2}} a_{1} e^{-\theta_{1}\left(1-x_{1}\right)} d x_{1} \\
& =\frac{2 a_{1} f_{1}\left(e^{\theta_{1}\left(q_{r}-1\right)}-e^{\theta_{1}\left(e_{1}-1\right)}\right)}{\theta_{1}\left(f_{1}-e_{1}\right)^{2}}+\frac{2 a_{1}\left(\left(e_{1} \theta_{1}-1\right) e^{\theta_{1}\left(e_{1}-1\right)}-\left(q_{r} \theta_{1}-1\right) e^{\theta_{1}\left(q_{r}-1\right)}\right)}{\theta_{1}^{2}\left(f_{1}-e_{1}\right)^{2}} \\
E\left(p_{s p}\right) & =\int_{0}^{q_{p}} f\left(x_{2}\right) a_{2} e^{-\theta_{2}\left(1-x_{2}\right)} d x_{2}=\int_{e_{2}}^{q_{p}} \frac{2\left(f_{2}-x_{2}\right)}{\left(f_{2}-e_{2}\right)^{2}} a_{2} e^{-\theta_{2}\left(1-x_{2}\right)} d x_{2} \\
& =\frac{2 a_{2} f_{2}\left(e^{\theta_{2}\left(q_{p}-1\right)}-e^{\theta_{2}\left(e_{2}-1\right)}\right)}{\theta_{2}\left(f_{2}-e_{2}\right)^{2}}+\frac{2 a_{2}\left(\left(e_{2} \theta_{2}-1\right) e^{\theta_{2}\left(e_{2}-1\right)}-\left(q_{p} \theta_{2}-1\right) e^{\theta_{2}\left(q_{p}-1\right)}\right)}{\theta_{2}^{2}\left(f_{2}-e_{2}\right)^{2}}
\end{aligned}
$$

